\title{
Membrane-associated Hsp72 from tumor-derived exosomes mediates STAT3-dependent immunosuppressive function of mouse and human myeloid-derived suppressor cells
}

Fanny Chalmin,, 1,2,3 Sylvain Ladoire,1,2,3,4 Grégoire Mignot, 1,2 Julie Vincent, 1,2,3 Mélanie Bruchard,1,2,3 Jean-Paul Remy-Martin, ${ }^{5}$ Wilfrid Boireau, ${ }^{6}$ Alain Rouleau, ${ }^{6}$ Benoit Simon, ${ }^{6}$ David Lanneau, ${ }^{1,3}$ Aurélie De Thonel,,1,3 Gabriele Multhoff, ${ }^{7}$ Arlette Hamman, ${ }^{1}$ François Martin, 1,2,3

Bruno Chauffert, 1,2,3,4 Eric Solary, ${ }^{1,3}$ Laurence Zitvogel,8 Carmen Garrido, ${ }^{1,3}$ Bernhard Ryffel, ${ }^{9}$ Christophe Borg, ${ }^{5}$ Lionel Apetoh, ${ }^{10}$ Cédric Rébé, ${ }^{1,2,3,4}$ and François Ghiringhelli1,2,3,4

\begin{abstract}
${ }^{1}$ INSERM U866, Dijon, France. ${ }^{2}$ INSERM AVENIR Team, Dijon, France. ${ }^{3}$ Faculty of Medicine and Pharmacy, University of Burgundy, Dijon, France. ${ }^{4}$ Anti-Cancer Centre Georges François Leclerc, Dijon, France. 5INSERM U645, EFS Bourgogne-Franche Comté, University of Franche-Comté, Besançon, France. ${ }^{6}$ FEMTO-ST Institute, University of Franche Comté, Besançon, France. ${ }^{7}$ Department of Radiation Oncology, University Hospital, Klinikum rechts der Isar, Technische Universität München, Munich, Germany. ${ }^{8}$ INSERM U805, Institut Gustave Roussy, Villejuif, France. 9Laboratory of Molecular Immunology and Embryology, CNRS UMR6218, Orléans, France. ${ }^{10} \mathrm{Center}$ for Neurologic Diseases,
\end{abstract} Brigham and Women's Hospital, Harvard Medical School, Boston, Massachusetts, USA.

\begin{abstract}
Myeloid-derived suppressor cells (MDSCs) have been identified in humans and mice as a population of immature myeloid cells with the ability to suppress $T$ cell activation. They accumulate in tumor-bearing mice and humans and have been shown to contribute to cancer development. Here, we have isolated tumor-derived exosomes (TDEs) from mouse cell lines and shown that an interaction between TDE-associated Hsp72 and MDSCs determines the suppressive activity of the MDSCs via activation of Stat3. In addition, tumor-derived soluble factors triggered MDSC expansion via activation of Erk. TDE-associated Hsp72 triggered Stat3 activation in MDSCs in a TLR2/MyD88-dependent manner through autocrine production of IL-6. Importantly, decreasing exosome production using dimethyl amiloride enhanced the in vivo antitumor efficacy of the chemotherapeutic drug cyclophosphamide in 3 different mouse tumor models. We also demonstrated that this mechanism is relevant in cancer patients, as TDEs from a human tumor cell line activated human MDSCs and triggered their suppressive function in an Hsp72/TLR2-dependent manner. Further, MDSCs from cancer patients treated with amiloride, a drug used to treat high blood pressure that also inhibits exosome formation, exhibited reduced suppressor functions. Collectively, our findings show in both mice and humans that Hsp72 expressed at the surface of TDEs restrains tumor immune surveillance by promoting MDSC suppressive functions.
\end{abstract}

\section{Introduction}

Myeloid-derived suppressor cells (MDSCs) have been identified in humans and mice as a population of immature myeloid cells with the ability to suppress $\mathrm{T}$ cell activation (1). In mice, MDSCs are uniformly characterized by the expression of the cell-surface antigens Ly-6C/G and CD11b (2), while in humans, MDSCs are typically $\mathrm{CD} 11 \mathrm{~b}^{+} \mathrm{CD} 33^{+} \mathrm{HLA}-\mathrm{DR} \mathrm{R}^{-}(3-6)$. In tumor-bearing mice, these cells have been shown to markedly expand systemically when mice are inoculated with transplantable tumor cells or when tumors spontaneously develop in transgenic mice with tissue-restricted oncogene expression (7). In addition, an increased MDSC frequency was detected in the blood of patients with different types of cancers $(4,8-10)$. In mice and humans, MDSCs from tumor bearers induce antigen-specific MHC class I-restricted tolerance of

Authorship note: Fanny Chalmin, Sylvain Ladoire, and Grégoire Mignot contributed equally to this work.

Conflict of interest: The authors have declared that no conflict of interest exists. Citation for this article: J Clin Invest. 2010;120(2):457-471. doi:10.1172/JCI40483.
$\mathrm{CD}^{+} \mathrm{T}$ cells (11) and are one of the major suppressors of antitumor immunity. Given that MDSCs from naive mice were generally found to lack immunosuppressive properties, it has been proposed that MDSCs require activation signals from tumor cells to support their suppressive function on T cells (12).

Recent evidence suggests that the transcriptional factor Stat3 is constitutively activated in many mouse and human cancer cells. Activated Stat3 is not only involved in tumor cell survival but has also been proposed to be the main regulator of MDSC expansion (13-15). Indeed, tumor cells that constitutively express tyrosine 705-phosphorylated Stat3 (tyrosine 705-pStat3) were shown to release tumor-derived factors that induce MDSC accumulation (13, 16-19). However, these observations were challenged by the report of Kortylewski et al., in which the specific deletion of Stat 3 in hematopoietic cells enhanced the presence of MDSCs in the tumor bed (20). Therefore, the exact role for Stat 3 within MDSCs remains elusive.

Tumor-induced activation and expansion of MDSCs can be mediated by the release of soluble factors but also by microvesicles known as exosomes $(21,22)$. These microvesicles are endo- 
some-derived organelles of 50 to $150 \mathrm{~nm}$ in size, which are actively secreted through an exocytosis pathway used in cells under normal as well as pathologic conditions for receptor discharge and intercellular crosstalk (23). While tumor-derived exosomes (TDEs) were initially described to be immunostimulatory, recent reports have shown that they could induce MDSC expansion (24) or inhibit T cell function or dendritic cell differentiation (25).

While several groups have studied the role of tumor-derived factors accounting for MDSC expansion, the mechanisms dictating their immunosuppressive activity in vivo have not been fully addressed. Given the key importance of Stat 3 in mediating immunosuppression, we assumed that Stat3, rather than mediating MDSC expansion, is actually responsible for the promotion of MDSC suppressive properties.

In this study, we report, using 3 different tumor cell lines, that TDEs triggered Stat 3 activation and MDSC suppressive activity without inducing their expansion. In sharp contrast, while tumor soluble factors devoid of exosomes were indeed able to induce MDSC expansion, they did not trigger Stat 3 activation and MDSC immunosuppressive functions. Mechanistically, we show in both mice and humans that Hsp72 expressed on exosome surface triggers Stat3 activation in MDSCs in a TLR2/MyD88-dependent manner through an autocrine production of IL-6. Targeting exosome production in vivo using dimethyl amiloride blunts the suppressive activity of MDSCs and enhances the efficacy of cyclophosphamide treatment in 3 different mouse tumor models. Dampening exosome production also diminishes immunosuppression in cancer patients. Altogether, our findings indicate that the immunosuppressive effect of tumor cells involves their capability of inducing functional MDSCs by releasing Hsp72expressing exosomes.

\section{Results}

Tumor exosome release promotes Stat3 activation in MDSCs. We determined whether the activation of MDSC suppressive functions was mediated by tumor-derived soluble factors (TDSFs) or TDEs, both contained in the tumor cell supernatant (TCS) in 3 mouse tumor cell lines (EL4 thymoma, TS/A mammary carcinoma, and CT26 colon carcinoma), that release equivalent exosome quantities in culture medium (Supplemental Figure 1; supplemental material available online with this article; doi:10.1172/JCI40483DS1).

Importantly, we noted a complete dissociation between TDSF and TDE properties. TDSFs induce MDSC expansion through proliferation of myeloid precursors (Figure 1, A and B), while TDEs drive Stat 3 phosphorylation (Figure 1C).

Stat 3 activation by TDEs and not by TDSFs was also observed when using MDSCs purified from naive mice (Supplemental Figure 2). Moreover, TDEs could trigger Stat3 phosphorylation in a dose-dependent manner in purified MDSCs isolated from the spleen of naive mice (Figure 1D).

In order to better characterize the discrepant effects of TDEs and TDSFs in the biology of MDSCs, we then investigated the transduction signaling pathways activated by TDEs and TDSFs within MDSCs. In line with our previous results, stimulation of MDSCs with TDSFs induced an activation of Erk without an activation of Stat 3 while TDEs only activated Stat 3 (Figure 1E). Interestingly, we found that TDSFs contained some GM-CSF (Figure 1F) and that the addition of anti-GM-CSF blunted the effect of TDSFs on Erk activation (Figure 1G) and MDSC expansion (Supplemental
Figure 3). We also studied NF-אB activation and found that TDEs induced a short but strong p 65 activation while TDSFs induced a prolonged but modest p65 activation (Supplemental Figure 4).

Altogether, we demonstrated that TCS triggers 2 distinct molecular pathways in MDSCs. TDSFs trigger the activation of Erk, which results in the expansion of MDSCs, while TDEs trigger the activation of Stat 3 without promoting MDSC expansion.

TDE-induced Stat 3 activation determines MDSC suppressive functions. Since our results suggested that Stat 3 was not involved in MDSC expansion, we sought to determine whether Stat 3 was involved in MDSC immunosuppressive functions. First, we showed that only MDSCs from tumor-bearing mice harbored pStat3 (Figure 2A), with a higher level in monocytic ones (Supplemental Figure 5). MDSCs from tumor-bearing mice also exerted a significant immunosuppressive effect, while MDSCs from naive mice did not express Stat 3 and did not have significant immunosuppressive functions (Figure 2B). In addition, Stat 3 inhibition using selective inhibitors or siRNA blunted MDSC immunosuppressive effect in vitro (Figure 2C). Also, in vivo adoptive transfer of MDSCs from tumor-bearing mice could annihilate the effect of a tumor vaccine on the occurrence of lung metastases in a pStat3-dependent manner (Figure 2D). Importantly, the MDSC adoptive transfer had no effect on lung metastasis growth in unvaccinated mice or nude mice, thus confirming that this effect is dependent on T cells.

Finally, only MDSCs incubated in the presence of TDEs - but not those incubated in the presence of TDSFs - exerted suppressive functions on antigen-stimulated OT-1 T lymphocytes in vitro (Figure $2 \mathrm{E}$ ) or were able to blunt IFN- $\gamma$ production of tumor-specific CD8 and CD4 lymphocytes in the spleen of tumor-bearing mice in vivo (Figure $2 \mathrm{~F}$ ).

Altogether, these data demonstrate that TDEs through their capacity to activate Stat 3 could mediate T cell-dependent immunosuppressive functions of MDSCs.

TDEs trigger pStat 3 expression in MDSCs through production of IL-6. We then sought to determine the mechanisms triggering Stat3 activation. In TCS, we could not detect any presence of classical Stat3-activating factors such as SCF, PGE2, IL-10, and IL-6 (Supplemental Figure 6A). We could not detect significant levels of PGE2 in either TCSs or TDEs (Supplemental Figure 6B). We observed that incubation of purified MDSCs from the spleen of naive mice with TDEs - but not with TDSFs - induced the production of IL-6, a known Stat3 activator (26) (Figure 3A). Interestingly, besides IL-6, none of the already described Stat3-activating cytokines was detected (Supplemental Figure 7). To assess the role of IL- 6 on Stat 3 activation, we demonstrated that culture of MDSCs from naive mice with either recombinant IL-6 (rIL-6) or TDEs induced Stat3 phosphorylation (Figure 3B). The addition of blocking anti-IL-6 Ab to TDEs in MDSC culture completely blocked Stat 3 phosphorylation, thus suggesting that IL-6 acts in an autocrine manner. To assess the in vivo relevance of this observation, we treated naive mice with 1 i.v. injection of either PBS, rIL-6, or TDEs. We observed that rIL- 6 or TDEs induced similar levels of pStat 3 in splenic MDSCs compared with the PBS control. In tumor-bearing mice, splenic MDSCs also harbored pStat 3 and a single injection of IL- 6 siRNA abolished this phosphorylation, thus demonstrating IL- 6 dependence of pStat 3 induction in MDSCs (Figure 3C).

In order to decrease the level of TDE release in tumor-bearing mice and its effect on IL-6-induced Stat 3 activation, we used dimethyl amiloride (27), an inhibitor of the $\mathrm{H}^{+} / \mathrm{Na}^{+}$and $\mathrm{Na}^{+} / \mathrm{Ca}^{2+}$ 

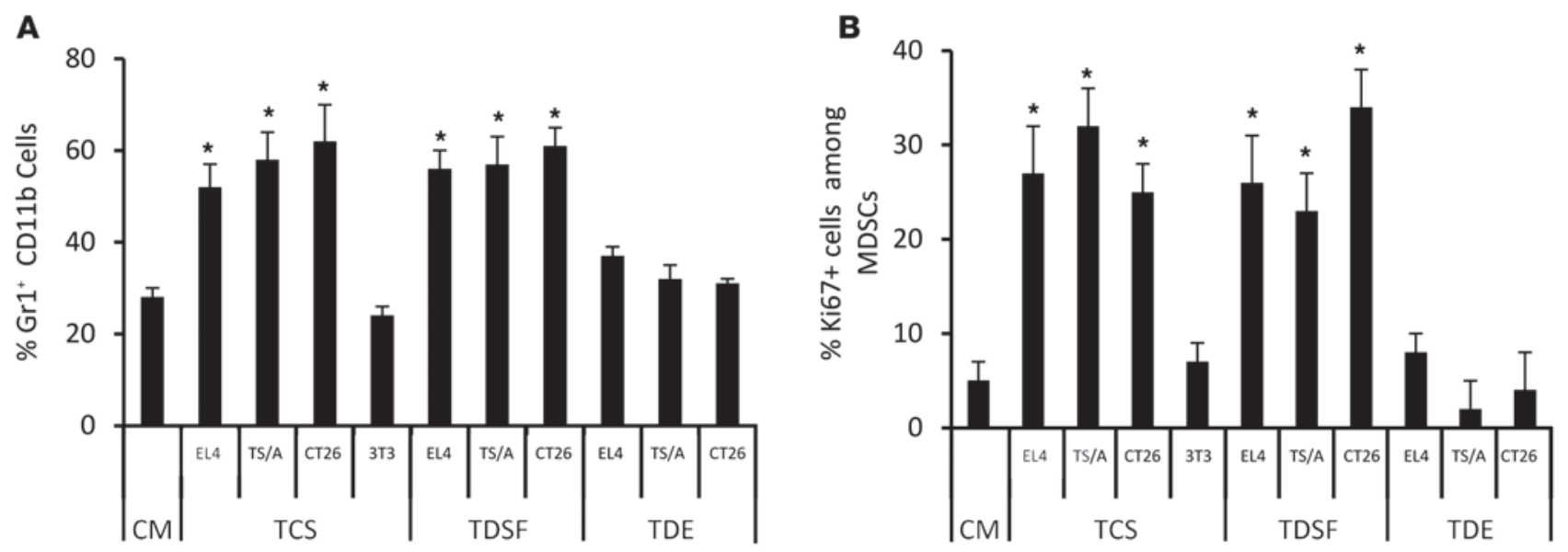

C
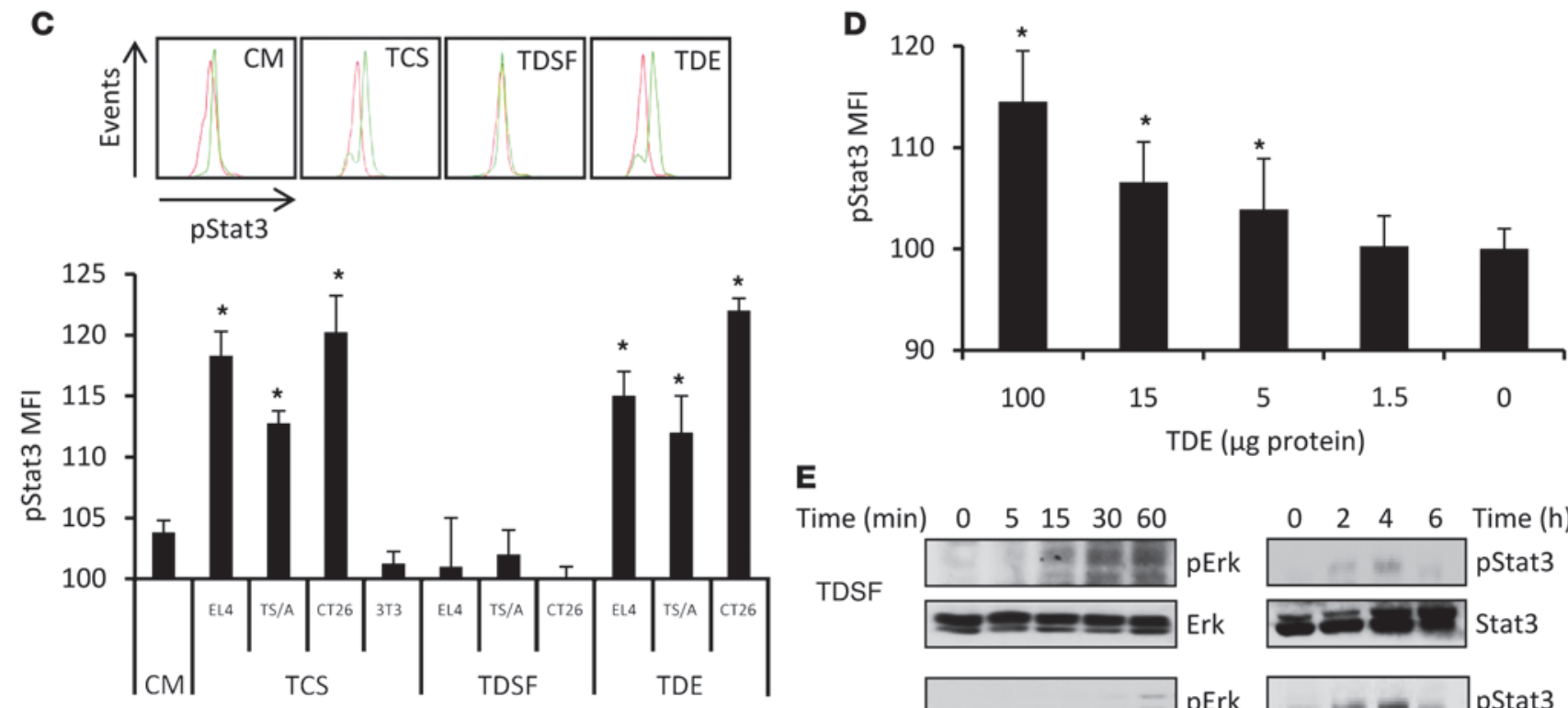

E
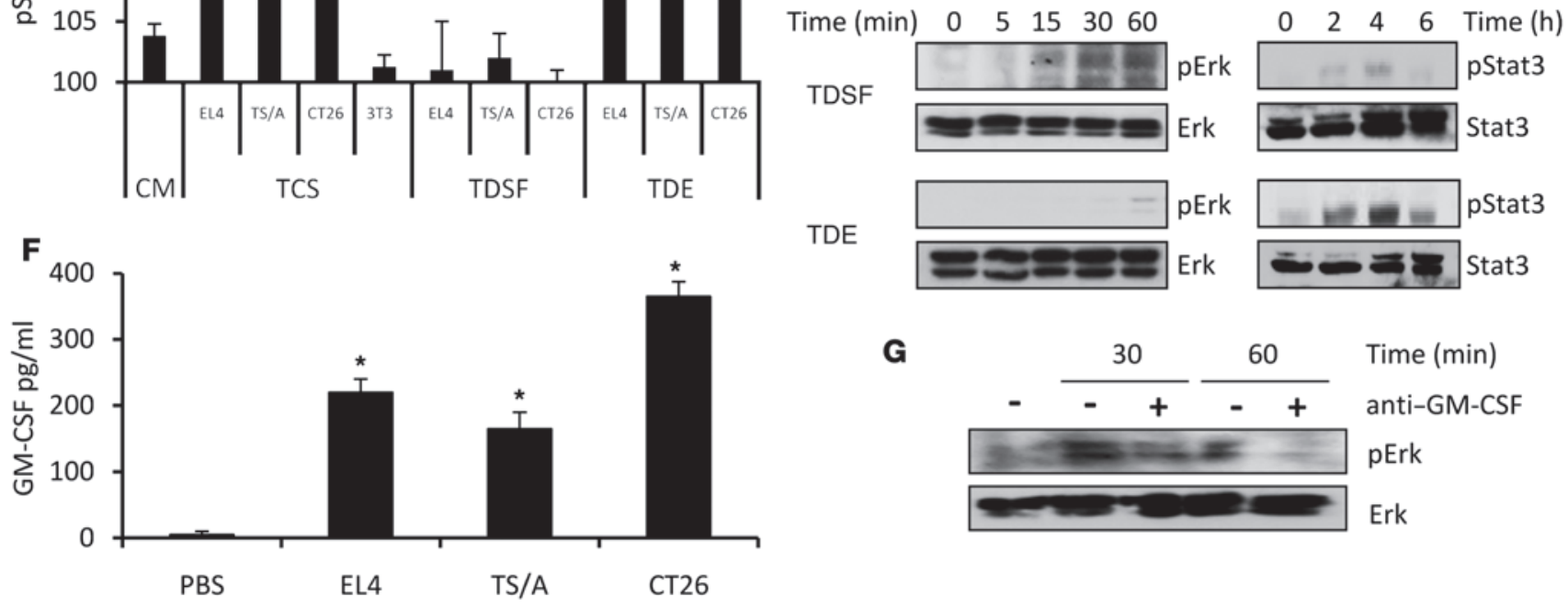

$\mathbf{G}$

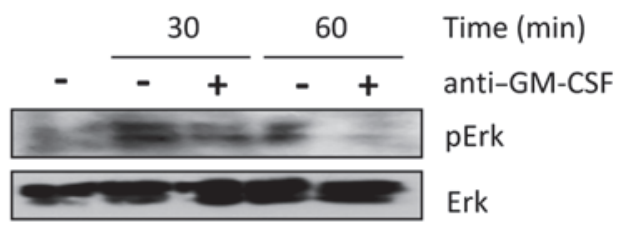

Figure 1

TDEs determine STAT3 activation, while TDSFs determine MDSC expansion. Bone marrow from naive mice was cultured 3 days in complete medium (CM) alone or with TCSs, TDEs, or TDSFs. (A) The percentage of Gr1+CD11 b+ precursor cells \pm SD was determined by flow cytometry. (B) The percentage of Ki67+ cells \pm SD in $\mathrm{Gr} 1{ }^{+} \mathrm{CD} 11 \mathrm{~b}^{+}$cells was determined by flow cytometry. (C) pStat3 MFI in Gr1+CD11b+ cells was determined by flow cytometry. Data represent MFI $\pm \operatorname{SD}(n=3)$; inset shows representative FACS histogram. (D) Purified splenic MDSCs from naive mice were treated with increasing dosages of TDEs. pStat3 expression was determined by FACS analysis. Data represent MFI \pm SD $(n=3)$. (E) Activation of Stat3 and Erk in MDSC clones stimulated by TDEs or TDSFs was determined by Western blotting. (F) GM-CSF production by tumor cells was determined by ELISA. (G) Activation of Erk by TDSFs plus anti-GM-CSF blocking Ab was assessed by Western blotting. ${ }^{*} P<0.05$. 
A
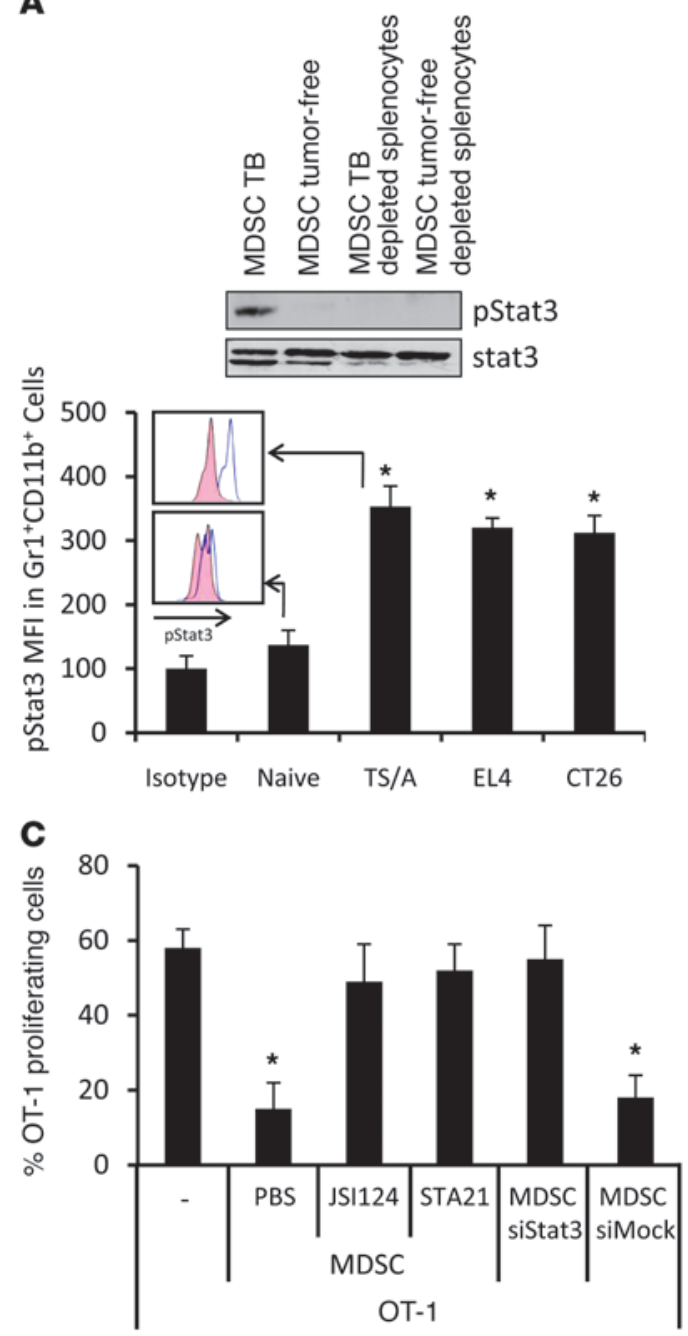

E

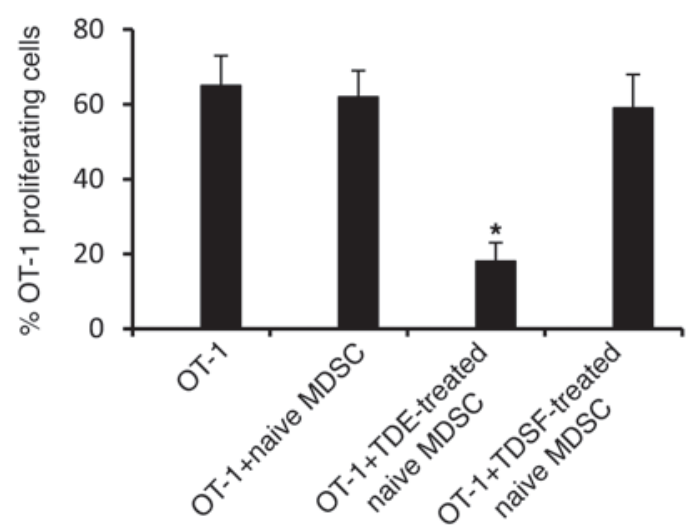

B
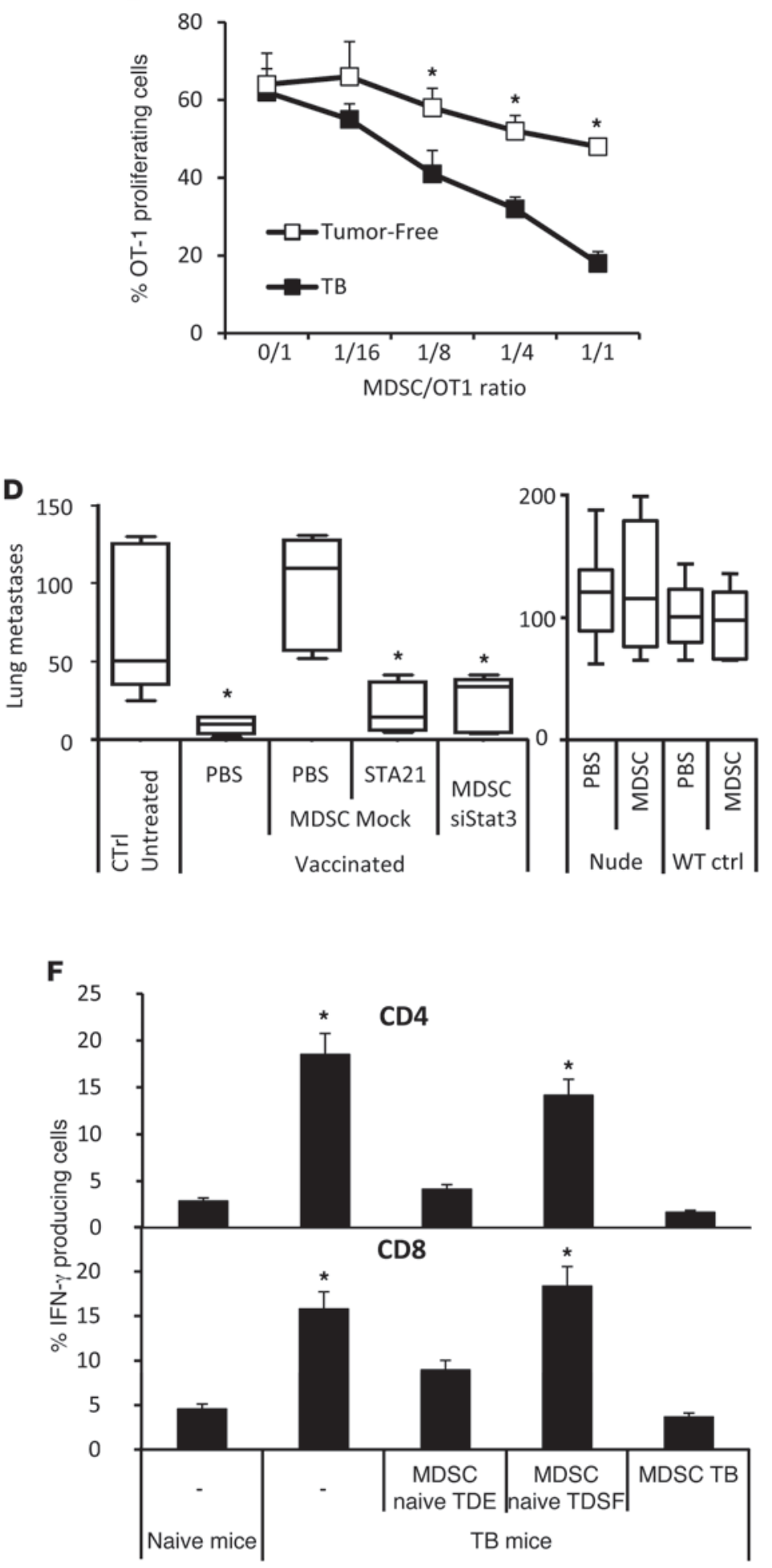


\section{Figure 2}

TDE-induced Stat3 activation determines MDSC suppressive functions. (A) Expression of pStat3 in MDSCs from naive and tumor-bearing mice (TB) was determined by Western blotting (upper panel) and FACS (lower panel). (B) CFSE-labeled OT-1 cells loaded with SIINFEKL and cultured alone or with MDSCs isolated from TB or naive mice at different MDSC/OT-1 ratios. Percentage of OT-1 proliferating cells was determined by flow cytometry $(n=3)$. (C) Peptide-loaded, CFSElabeled OT-1 cells were cultured alone or with MDSCs isolated from TB mice treated with PBS, JSI124, STA21, or Stat3 siRNA. CFSE dilution was determined by flow cytometry $(n=3)$. (D) Nude or WT mice were vaccinated or not with frozen/thawed CT26 cells 1 week before i.v. injection of CT26 cells admixed or not with MDSCs isolated from TB and previously treated with STA21 or Stat3 siRNA. 2 weeks later, lung metastasis numbers were evaluated ( $n=5$ mice per group). For box and whisker plots, bottoms and tops of boxes show the 25th and 75th percentiles, respectively, and middle bands show the median; whiskers show extrema. (E) Peptide-loaded, CFSE-labeled OT-1 cells were cultured alone or with bone marrow-derived MDSCs of naive mice previously treated alone or with tumor cell whole supernatant or TDE or TDSF fractions. CFSE dilution was determined by flow cytometry ( $n=3$ mice per group). (F) TB mice were injected or not with MDSCs. These MDSCs were either from TB or naive mice and stimulated with PBS, TDEs, or TDSFs. 2 days later, spleen cells were harvested and restimulated in vitro with $\mathrm{CD} 3 \mathrm{mAb} \mathrm{b}^{+}$dead tumor cells, then stained for intracellular CD4 and IFN- $\gamma$ (upper panel) or CD8 IFN- $\gamma$ (lower panel). ${ }^{*} P<0.05$. Error bars represent mean + SD.

channels, or omeprazole, a $\mathrm{K}^{+} / \mathrm{H}^{+}$ATPase inhibitor. Both have been previously involved in exosome release $(28,29)$. In vitro exposure of tumor cells to dimethyl amiloride (DMA) or omeprazole and in vivo treatment of tumor-bearing mice with DMA or omeprazole reduced exosome release in culture medium and blood serum, respectively (Figure 3D). In vivo, while TDE injection induces IL-6 in sera of naive mice, DMA or omeprazole treatment decreased IL-6 serum levels in tumor-bearing mice (Figure 3E). Moreover, DMA or omeprazole treatment abolished pStat 3 in MDSCs of tumorbearing mice, as IL-6 siRNA injection did (Figure 3F). Finally, treatment of tumor-bearing mice with DMA abrogated the immunosuppressive effect of adoptively transferred MDSCs on the antitumor activity of a tumor vaccine (Figure 3G).

Altogether, these results indicate that TDEs trigger an IL-6dependent acquisition of pStat 3 on MDSC. DMA or omeprazole, by reducing exosome production, could abrogate in vivo Stat3 phosphorylation in MDSCs and their suppressor activity.

IL-6-induced pStat3 expression in MDSCs is TLR2-MyD88 dependent. Extracellular microbial components are recognized by TLRs that stimulate inflammation, but some mammalian cellular components can also stimulate TLRs (30), raising the possibility that these receptors have a role in response to danger signals released from tumor cells (31). TLRs are also the main inducers of IL-6 in myeloid cells (32). We thus explored the putative involvement of TLR family members in sensing TDEs. Splenic MDSCs from naive WT mice and mice deficient in TLR2, TLR4, or their adaptor proteins, MyD88 and TIR domain-containing adaptor-inducing interferon $\beta$ (Trif), were examined for IL- 6 production after stimulation with TDEs. TDE-induced IL- 6 was fully dependent on TLR2 and MyD88 but not on TLR4 or Trif (Figure 4A). Similarly, Stat3 phosphorylation induced by TDEs was also fully dependent on TLR2 and MyD88 (Figure 4B). These observations were not biased by a different intrinsic capacity of transgenic mice to produce IL-6 or to activate Stat3 (Supplemental Figure 8). During EL4 tumor growth, $\mathrm{Gr}-1^{+} \mathrm{CD} 11 \mathrm{~b}^{+}$cells accumulated at comparable levels in the spleen of WT and TLR signaling-deficient mice (Figure 4C). In contrast, pStat 3 expression was fully dependent on TLR2 and MyD88 (Figure 4C). Moreover, MDSCs from tumor-bearing TLR2deficient mice exhibited a drastically lower capacity to inhibit antigen-specific OT-1 proliferation than MDSCs from WT tumorbearing mice (Figure 4D). Interestingly, EL4 growth was slightly but significantly decreased in TLR2-deficient mice (Figure 4E).

Altogether, these results demonstrate that in vitro and in vivo TDEs induce IL- 6 production by MDSCs through activation of TLR2 and its adaptor MyD88, leading to Stat 3 phosphorylation and the promotion of MDSC immunosuppressive functions.

Stat 3 activation in MDSCs is dependent on Hsp72 expressed at the surface of TDEs. To identify the factor present on TDEs that could induce Stat3 phosphorylation and MDSC activation, we first screened the expression of known endogenous TLR2 ligands in TDEs, TDSFs, and whole-tumor cells by immunoblotting (32-38). TDSFs did not express any TLR2 ligands. TDEs from the 3 cell lines expressed Hsp72 and Hsc73 but did not express other known endogenous TLR2 ligands (Figure 5A). To eliminate the potential role of other microparticles copurified with exosomes, we checked to determine that Hsp72 was only present in exosomal fractions, and not in other microparticles (Supplemental Figure 9). Using TDE-coated beads, we showed that Hsp72, but not Hsc73, was detected on TDE cell surface, while it was not expressed on the surface of 3T3 exosomes (Figure 5B). This was confirmed by electronic microscopy with immunogold labeling (not shown). A physical interaction between TLR2 and Hsp72 was studied by surface plasmon resonance (Figure 5C) and involved hydrophobic interactions. In vitro, $\mathrm{rHsp} 72$ added to MDSC culture could trigger pStat 3 expression and IL-6 production when MDSCs were obtained from WT mice but not from TLR2-deficient mice (Figure 5D and Supplemental Figure 10). Importantly, no production of TNF- $\alpha$ could be detected. At a molecular level, exposure of MDSCs to rHsp72 induced Stat 3 phosphorylation and Stat 3 and p65 transcriptional activity without activation of Erk (Supplemental Figure 11). IL-6-blocking Ab blunted rHsp72-dependent Stat3 phosphorylation, thus demonstrating that $\mathrm{rHsp} 72$ can trigger the TLR2-dependent IL-6/pStat3 pathway in MDSCs (Figure 5D). To eliminate the involvement of a potential contamination with endotoxins, we demonstrated that boiled Hsp72 could not induce IL-6 secretion and Stat3 phosphorylation in MDSCs; furthermore, we routinely tested for the absence of mycoplasms (Figure 5D and data not shown). Moreover, rHsp72 effect on pStat3 induction could be abrogated by adding an anti-Hsp72 Ab, while this treatment remained inefficient when pStat 3 was induced by a bacterial TLR2 ligand (PAM3CSK4) (Figure 5D).

To confirm the specific role of Hsp72 in TDEs, we stably transfected CT26 cells with Hsp72 shRNA. Two clones (called H96 and H97) with a reduced Hsp72 expression in cells and TDEs were selected (Supplemental Figure 12). Exosomes from mock-transfected CT2 6 cells induced Stat 3 phosphorylation and IL- 6 production in a TLR2-dependent manner, and this effect could be blunted by addition of a blocking anti-IL-6 Ab or an anti-Hsp72 Ab (Figure $6 \mathrm{~A}$ and data not shown). In contrast, TDEs obtained from $\mathrm{H} 96$ and H97 clones had a reduced capacity to trigger pStat 3 and IL-6 production in MDSCs compared with TDEs from mock CT26.

In vivo, a single i.v. injection of TDEs from mock-transfected CT26 cells but not from $\mathrm{H} 96$ or H97 cells induced pStat 3 expression in splenic MDSCs (Figure 6B). In a tumor setting, 15 days 


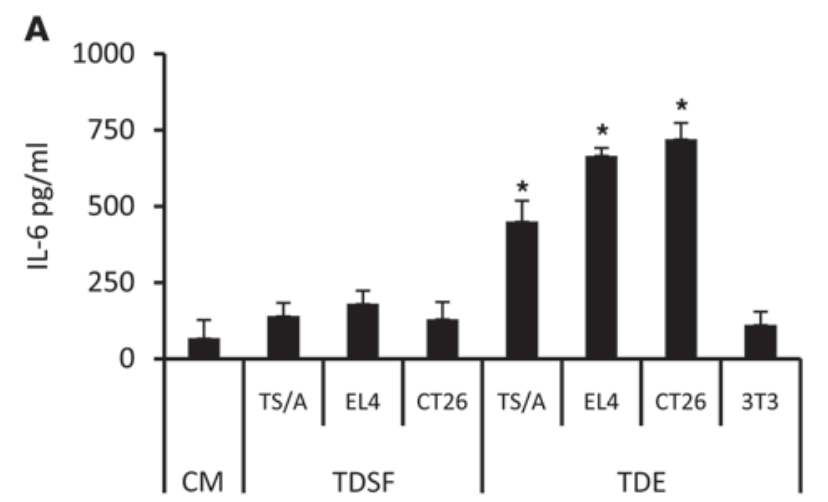

C
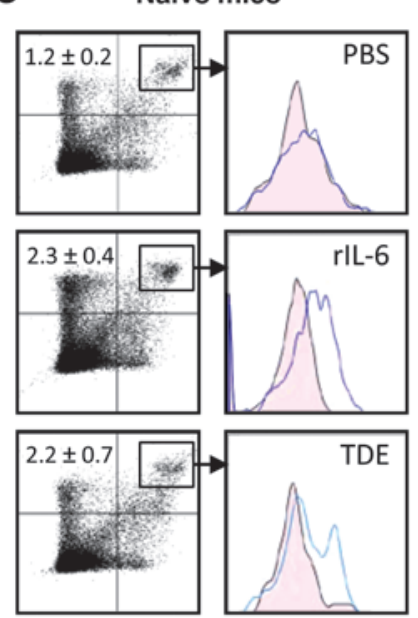

TB mice
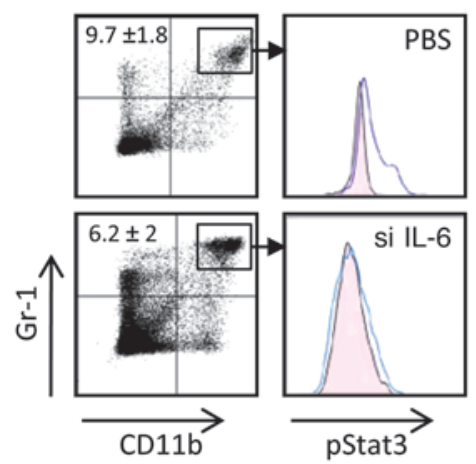

D
B
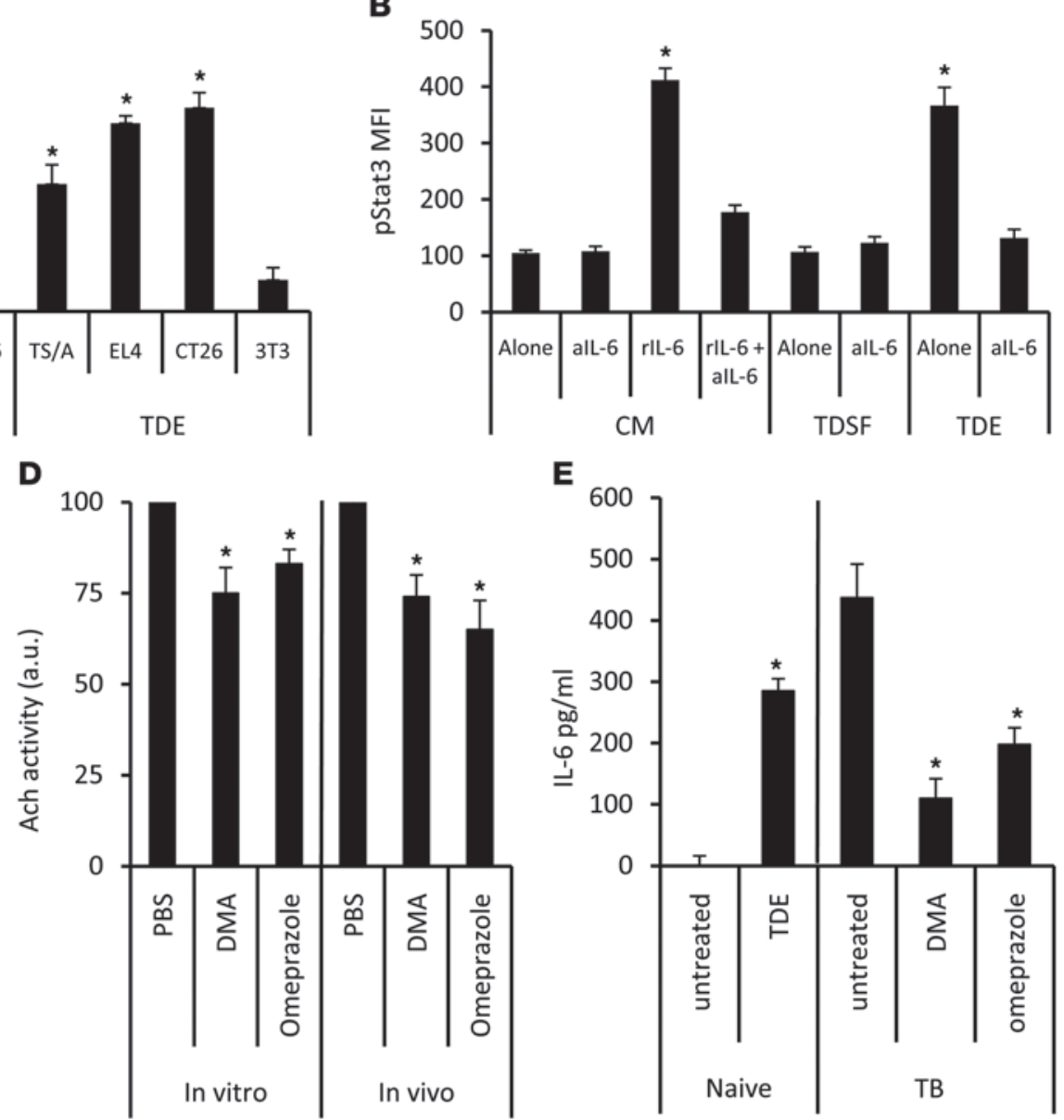

E
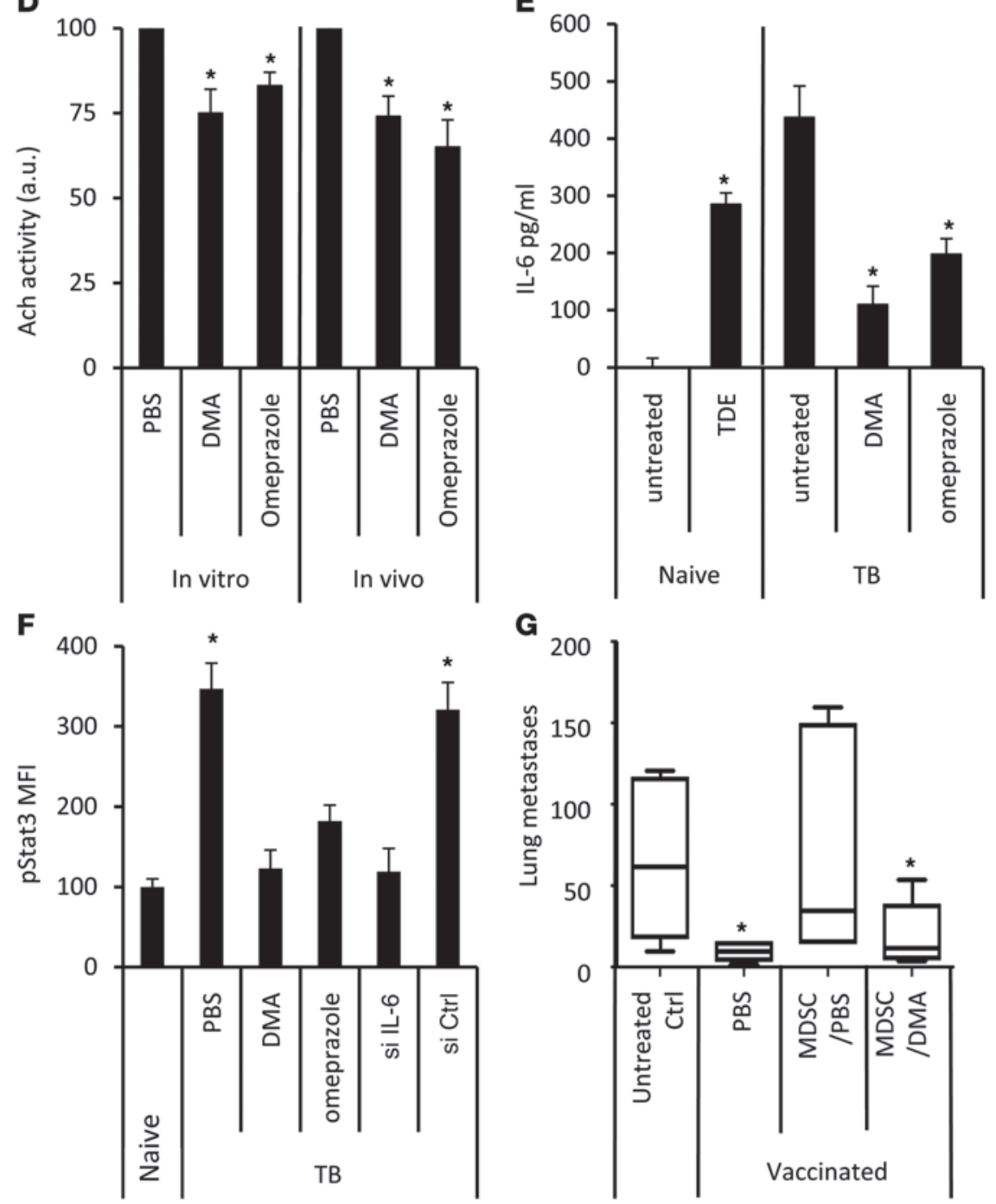


\section{Figure 3}

TDEs trigger pStat3 expression in MDSCs through autocrine production of IL-6. MDSCs from naive mice were treated with PBS or with TDSFs or TDEs. (A) IL-6 concentration in the supernatant was determined by ELISA. (B) In some wells, rIL-6 or blocking anti-IL-6 $\mathrm{mAb}$ (alL-6) were added. Data represent pStat3 MFI \pm SD. (C) Naive mice were i.v. injected with PBS, EL4 TDEs, or rIL-6. EL4 TB mice were i.v. injected with PBS or IL-6 siRNA. 24 hours later, spleens were harvested. MDSC percentage (denoted) and pStat3 expression were determined by FACS on gated MDSCs. (D) EL4 cells were cultured in vitro for 24 hours and treated with PBS, DMA, or omeprazole. In vivo EL4 TB mice were injected daily with DMA for 1 week. Acetylcholine esterase activity was assayed in supernatant or sera. (E) IL-6 concentration in sera was determined by ELISA in naive mice, which received a single injection of PBS or EL4 TDEs (left), or in EL4 TB mice, which received 1 daily injection of PBS, DMA, or omeprazole for 1 week (right). (F) EL4 TB mice were injected with PBS alone or DMA, omeprazole, or IL-6 siRNA (si). Spleens were harvested 24 hours later, and pStat3 expression was determined by FACS. Data are shown as $\mathrm{MFI} \pm \mathrm{SD}$. (G) Mice $(n=5)$ were vaccinated with frozen/thawed CT26 cells 1 week before i.v. injection of live CT26 admixed or not with MDSCs isolated from PBS- or DMA-treated CT26 TB mice. Twelve days later, lung metastasis number was evaluated. Each experiment was done in triplicate. For box and whisker plots, bottoms and tops of boxes show the 25th and 75th percentiles, respectively, and middle bands show the median; whiskers show extrema. ${ }^{*} P<0.05$.

after s.c. inoculation of mock-transfected CT26 or clones H96 or H97 transfected with Hsp72 shRNA, FACS analysis demonstrated that Hsp72 shRNA CT26 tumor-bearing mice harbored a drastically lower level of pStat 3 in splenic MDSCs, compared with MDSCs from mice bearing mock-transfected tumors (Figure 6C). Accordingly, adoptive transfer of MDSCs isolated from Hsp72 shRNA CT26 tumor-bearing mice have no significant immunosuppressive effect and could not abrogate the antitumor efficacy of a tumor vaccine in contrast to MDSCs from mock-transfected CT26 tumor-bearing mice (Figure 6D). To rule out a potential direct effect of IL- 6 produced by MDSCs on tumor cells, we showed that the transfection of tumor cells with siRNA gp130 (the IL-6R) did not inhibit the effect of MDSC adoptive transfer (not shown).

In conclusion, the immunosuppressive effects of MDSCs from tumor-bearing mice are induced by Hsp72 expressed at the surface of TDEs that triggers TLR2 signaling in MDSCs. This event induced an IL-6-dependent Stat3 phosphorylation within MDSCs and thus their immunosuppressive activity.

Exosome depletion by DMA restores the efficacy of cyclophosphamide by inhibiting MDSC functions. Given that TDEs are responsible for MDSC immunosuppressive function, we tested to determine whether DMA could restore the efficacy of cancer therapies by inducing exosome depletion. We previously demonstrated that the antitumor activity of low doses of the alkylating agent cyclophosphamide was related to its capability of inducing a T cell-dependent immune response through elimination of regulatory $\mathrm{T}$ cells rather than to its cytotoxic effect on tumor cells $(39,40)$. Cyclophosphamide reduces tumor growth and prolongs the survival of tumorbearing immunocompetent WT mice, yet is ineffective in athymic nude mice (Supplemental Figure 13). In our 3 tumor models, DMA alone had little or no effect, while the association of DMA with cyclophosphamide demonstrated a synergistic effect and drastically reduced tumor growth compared with cyclophosphamide alone (Figure 7A). A comparable synergic effect was observed with combination of DMA and another immunotherapy, intratumoral CPG injection (Figure 7B). Combined therapy (cyclophosphamide plus DMA) was ineffective in nude mice, underlining the critical role of $T$ cells for the efficacy of the combined treatment (Figure 7C).

To determine whether the antitumor effect of DMA was dependent on blocking the immunosuppressive function of MDSCs, we performed adoptive transfer of MDSCs from tumor-bearing mice after cyclophosphamide injection. Interestingly, transfer of MDSCs from tumor bearers completely abrogated the antitumor effect of DMA in the CT26 model (Figure 7D). In contrast, adoptive transfer of MDSCs from naive mice had no effect on tumor growth (not shown). Finally, to determine the role of tumor-derived Hsp72 in the therapeutic effect of DMA cyclophosphamide combination, we injected naive mice with mock-transfected or Hsp72 shRNA-transfected (clone H96 or H97) CT26 cells. As expected, we observed that, in mice bearing $\mathrm{H} 96$ or $\mathrm{H} 97$ tumors, cyclophosphamide alone had the same efficacy as cyclophosphamide plus DMA, demonstrating that the efficacy of DMA was strictly dependent on tumor Hsp72 expression by TDEs (Figure 7E).

Altogether, these data demonstrate that exosome depletion using DMA enhances the antitumor efficacy of cyclophosphamide. This synergic effect of DMA plus cyclophosphamide is dependent on $\mathrm{T}$ cells, MDSCs, and Hsp72.

Human TDEs dictate Stat 3 activation in MDSCs through TLR2 and Hsp72. Human MDSCs were phenotypically determined by the expression of the common myeloid marker CD33 and MHC class II (3-6). We observed as previously reported $(4,8-10,41)$ a significant increase in the frequency of MDSCs in the blood of metastatic cancer patients compared with healthy volunteers (Figure 8A). Consistent with the murine data, MDSCs from metastatic cancer patients exerted higher immunosuppressive function on $\mathrm{T}$ cells compared with MDSCs from healthy volunteers (Figure 8B).

In vitro, PBMCs from healthy volunteers were cultured 24 hours alone or in the presence of TDEs obtained from the $\mathrm{H} 23$ cell line, a lung adenocarcinoma that produced Hsp72-expressing TDEs (Supplemental Figure 14). H23 TDEs induced pStat 3 expression in MDSCs, and this effect was abolished by inhibition of TLR2 and Hsp72 (Figure 8C). Treatment of PBMCs from healthy volunteers by $\mathrm{H} 23$ exosomes strongly inhibited $\mathrm{T}$ cell proliferation after nonspecific $T$ cell triggering, and this immunosuppressive effect was significantly reduced after blockade of Hsp72 or TLR2 (Figure 8D).

DMA is not used in human therapy, but its analog amiloride, which exerts the same effect on exosome secretion (not shown), is currently used for treatment of edema or high blood pressure. In a series of 11 patients with colorectal metastatic cancer in whom amiloride was indicated for the treatment of high blood pressure, we obtained blood samples before and 3 weeks after the beginning of amiloride treatment and observed that amiloride reduced autologous serum capacity to induce phosphorylation of Stat3 in MDSCs (Figure 8E) and blunted MDSC suppressor functions (Figure 8F).

Altogether, these data demonstrated that human TDEs could activate human MDSCs and trigger their suppressive function in an Hsp72/TLR2-dependent manner. Moreover, blocking exosome formation in cancer patients with amiloride could possibly lead to the restoration of $\mathrm{T}$ cell functions.

\section{Discussion}

Our results identify a new mechanism that leads to an enhancement of tumor-associated MDSC immunosuppressive functions. First, we demonstrated that 2 distinct molecular pathways 
A

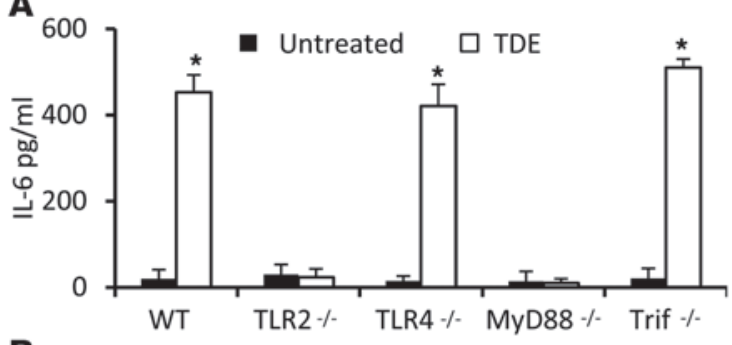

B
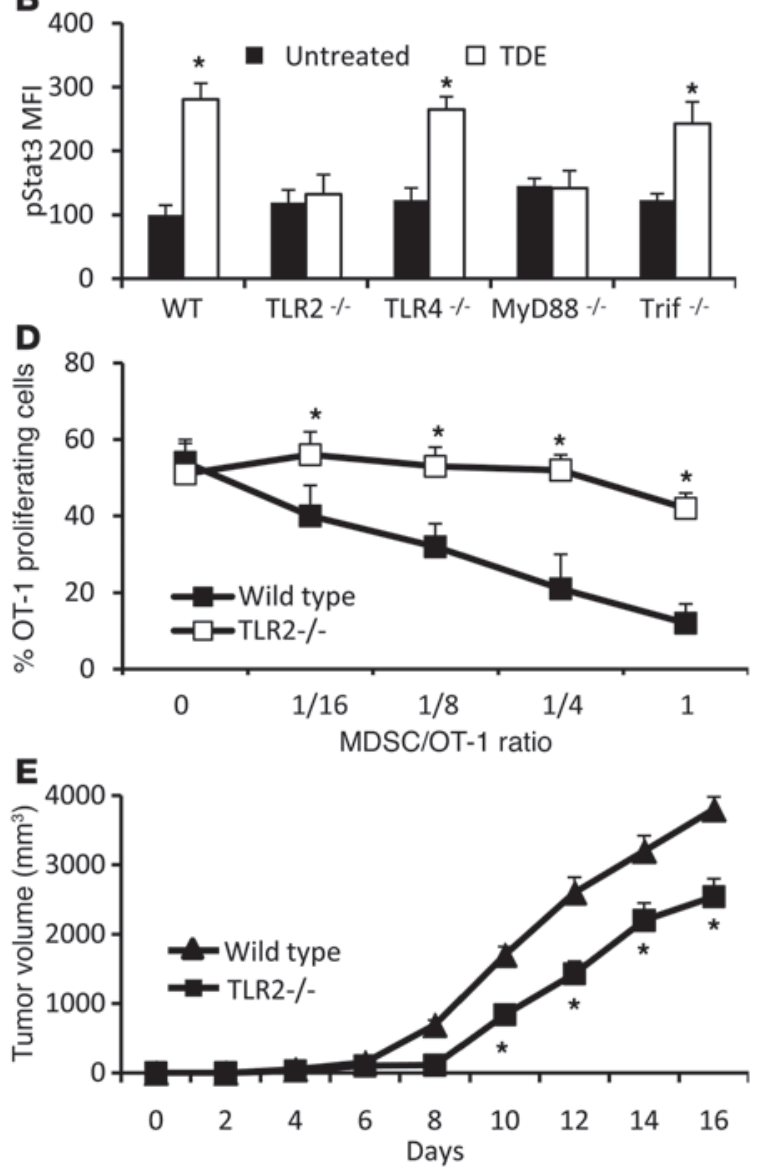

C
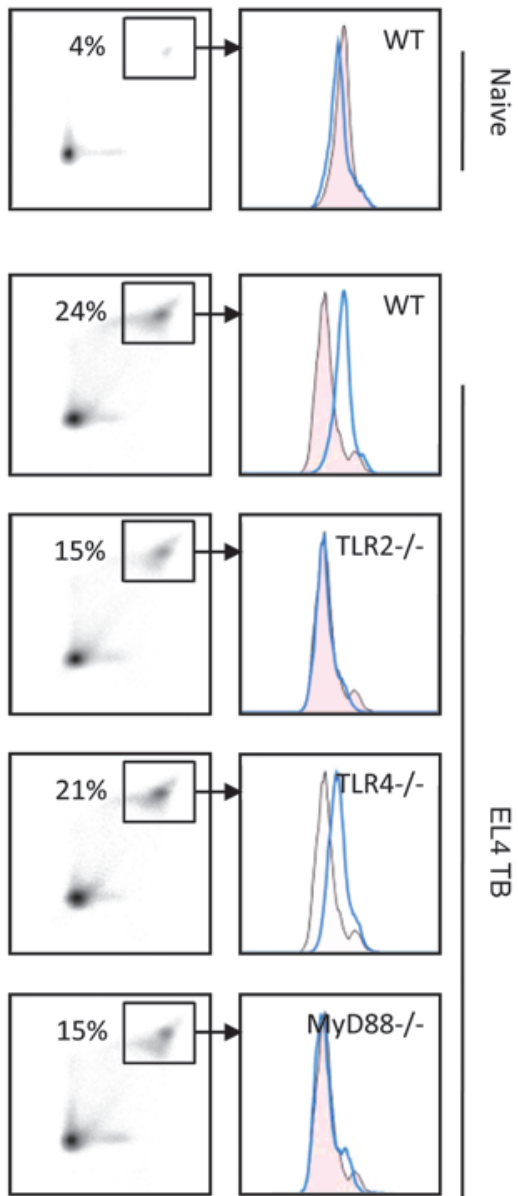

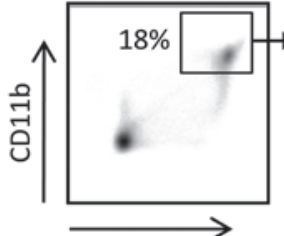

$\mathrm{Gr}-1$

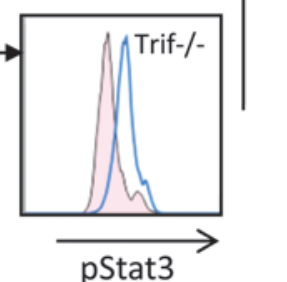

Figure 4

IL-6-induced pStat3 expression in MDSCs is dependent on the TLR2/MyD88 pathway. Purified MDSCs from WT or TLR2-, TLR4-, MyD88-, and Trif-deficient C57BL/6 tumor-free mice were cultured for 24 hours in complete medium supplemented or not with TDEs. IL-6 concentration in the supernatant was determined by ELISA (A), and pStat3 expression in cells was determined by FACS analysis (B). Data represent mean \pm SD. (C) WT C57BL/6 mice or TLR2-, TLR4-, MyD88-, and Trif-deficient mice were s.c. injected with $1 \times 10^{6}$ EL4 cells. 3 weeks later, spleen cells were harvested, MDSC percentage was determined in spleen (denoted in left panels), and pStat3 expression was determined by FACS analysis on MDSC gated cells (right panel). (D) $2 \times 10^{5}$ OT- 1 cells were labeled with CFSE, loaded with $10 \mu \mathrm{g} / \mathrm{ml}$ of SIINFEKL, and cultured 3 days alone or with different ratios of MDSCs from EL4 tumor-bearing WT mice or from EL4 tumor-bearing TLR2-deficient mice. Percentage of OT-1 proliferating cells was determined by flow cytometry. Each experiment was done in duplicate ( $n=3$ mice per group). (E) WT or TLR2 ${ }^{-/-}$mice were injected s.c. with EL4 cells, and tumor growth was monitored. ${ }^{*} P<0.05$.

account for MDSC expansion and activation. We then unraveled for what we believe is the first time that Stat3, rather than mediating MDSC expansion, actually promotes their immunosuppressive functions. At the molecular level, Hsp72 on TDEs, which binds to TLR2 on MDSCs, is the ligand that is responsible for their activation and the enhancement of their suppressive ability. Additionally, we showed that amiloride was able to interfere with our proposed mechanism leading to MDSC activation, thus restoring the efficacy of immunotherapy. We showed that our proposed mechanism leading to MDSC activation was also relevant in humans.

The literature clearly demonstrated that factors released by tumor cells could trigger Stat3 activation and expansion of MDSCs. Most reports suggested without clear demonstration that expansion of MDSCs may be due to Stat3 activation by tumorderived factors. However, Kortylewski et al. (20) challenged this 

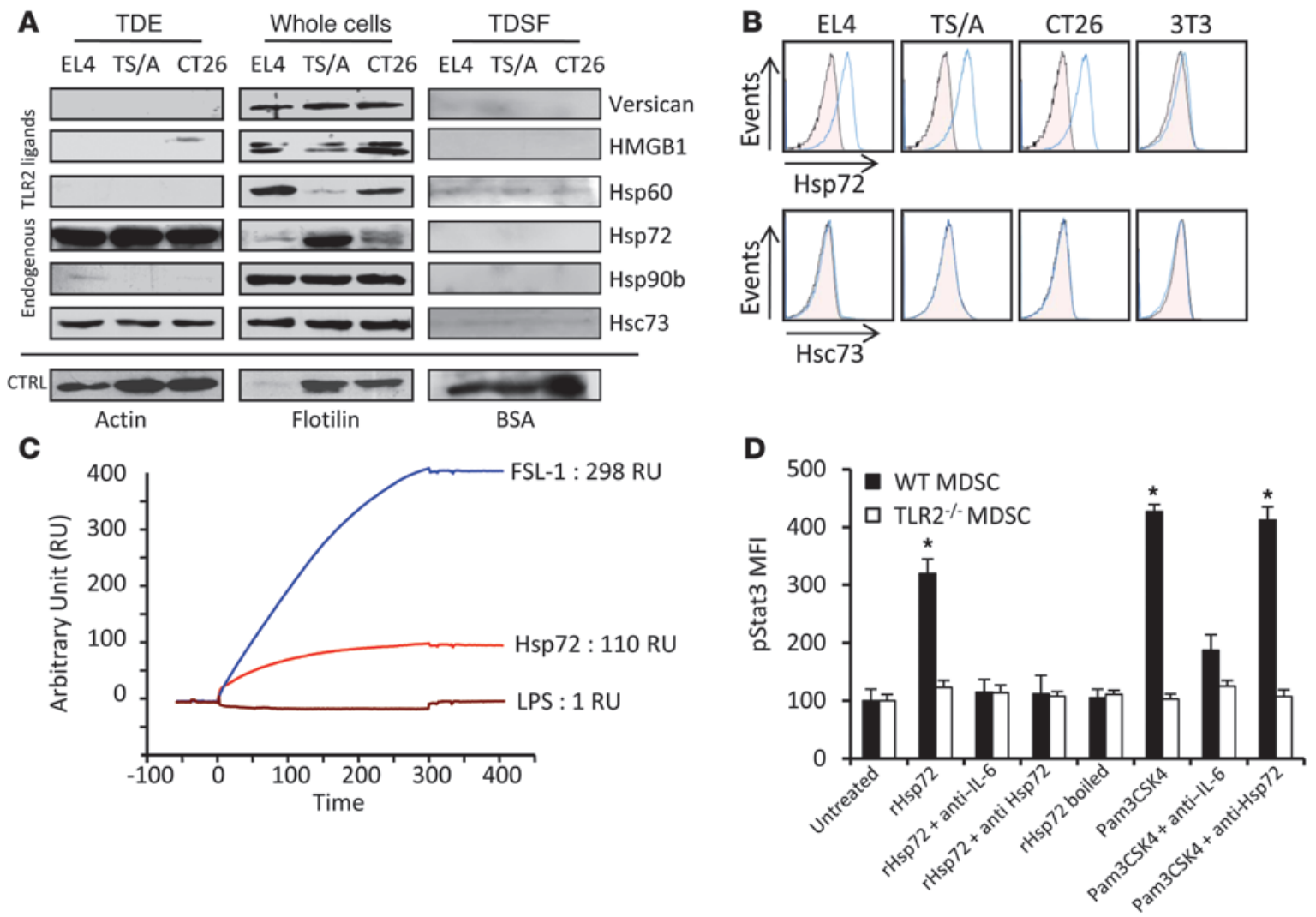

\section{Figure 5}

Hsp72 is expressed at the surface of TDEs and bound on TLR2. (A) Endogenous TLR2 ligand expression was investigated by immunoblots in TDEs, whole-cell lysates, and TDSFs from EL4, TS/A, and CT26 tumor cells. CTRL, control. (B) TDEs from EL4, TS/A, and CT26 tumor cells and exosomes from control 3T3 cells were coated on beads and labeled with control isotype or anti-Hsp72 or anti-Hsc73 Abs. Then FACS analysis was performed to determine expression of Hsp72 and Hsp73 at the surface of exosomes. One representative FACS histogram (blue line) is represented with its isotype control (pink area). (C) Surface plasmon resonance studying the binding of Hsp72 to TLR2 protein. FSL-1 (a bacterial TLR2 ligand) was used as positive control; LPS was used as negative control. (D) Purified myeloid cells from naive WT or TLR2-deficient C57BL/6 mice were cultured in complete medium supplemented or not as indicated. pStat3 expression was determined by FACS analysis on MDSC gated cells. Data represent MFI \pm SD. ${ }^{*} P<0.05$.

hypothesis by showing that, in mice with specific deletion of Stat 3 in hematopoietic cells, higher numbers of $\mathrm{Gr}^{+} \mathrm{CD}^{+} 1 \mathrm{~b}^{+}$ cells were found in the tumor bed. In the same line, other reports suggested in models where tumor cells could produce SCF that MDSC proliferation could be due to the activation of the SCF/Kit pathway $(42,43)$. Finally, the group from Ostrand-Rosenberg and a recent report from Xiang et al. demonstrated that PGE2 could be involved in MDSC activation and expansion $(24,44)$. Here, we provided evidence in 3 models in which neither SCF nor PGE2 were involved (Supplemental Figure 6) that Stat 3 is not linked to MDSC expansion but implicated in their activation (Figure 1). In contrast, GM-CSF produced by tumor cells is involved in MDSC expansion through the activation of the Erk pathway.

Exosomes are endosome-derived microvesicles that are notably secreted by the tumor cells in their environment. Interestingly, TDEs are a source of shared tumor rejection antigens and in some conditions could induce a $\mathrm{T}$ cell-dependent immunity in mice and human tumor models $(45,46)$. Therefore, injection of a high amount of TDEs could represent a source of tumor-rejection antigens relevant for immunointerventions (21). However, as TDEs retain a large part of the protein repertoire of tumor cells, they could also trigger immunosuppression. Indeed, crucial components of the immune response, such as dendritic cells, are profoundly affected by the encounter with TDEs (25). Some TDEs could also express factors such as Fas ligand that induce apoptosis of CD8 T cells and may be involved in immune evasion (47). In our study, we demonstrate for what we believe is the first time that TDEs have another immunosuppressive capacity, which is triggered by Stat 3 phosphorylation in MDSCs. Tumor-derived factors had been demonstrated to trigger MDSC accumulation from hematopoietic precursors $(8,48)$ and Stat 3 phosphorylation $(16$, 17), but the tumor factors involved in these effects remained to be discovered. Here, we unraveled the capacity of TDEs to induce Stat 3 phosphorylation and the acquisition of suppressive function in MDSCs (Figure 2). In a recent paper, Xiang et al. suggested that TDEs and exosome derived from tumor dissociation could induce MDSC expansion and Stat 3 activation, but they did not test the effect of the TDSFs. Moreover, the effect of TDEs is correlated to their concentration of PGE2 and blunted by PGE2 inhibition, thus leading to the hypothesis that TDE effect was mainly the effect of PGE2 contained in exosomes. In our models, we provided evidence that no PGE2 was present in TDEs or TDSFs. So we could con- 
A

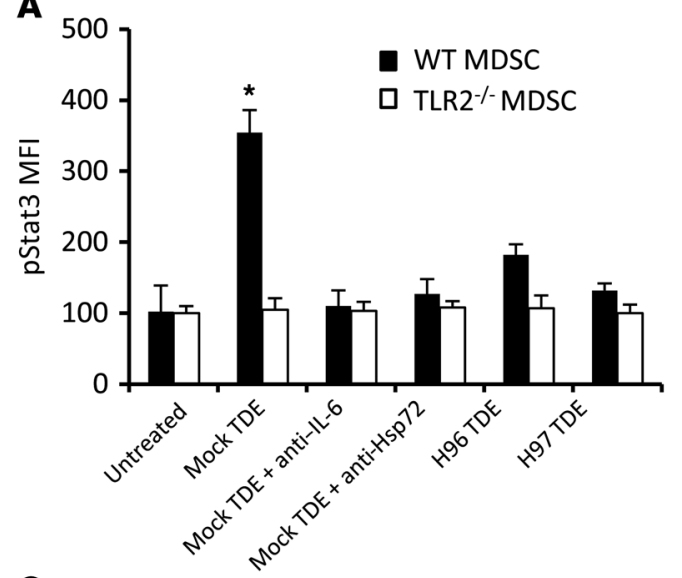

C

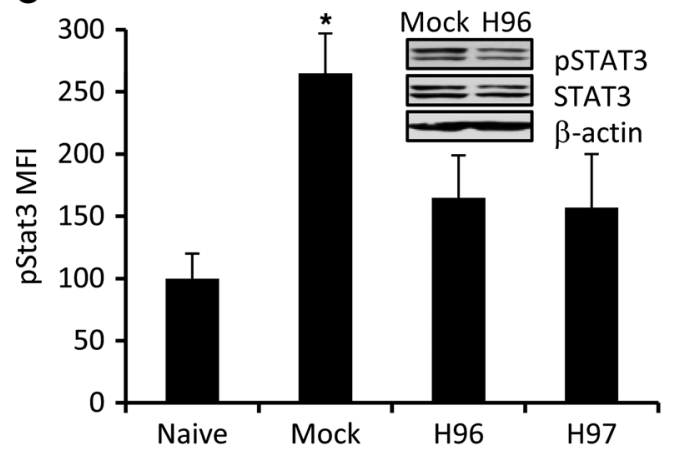

B

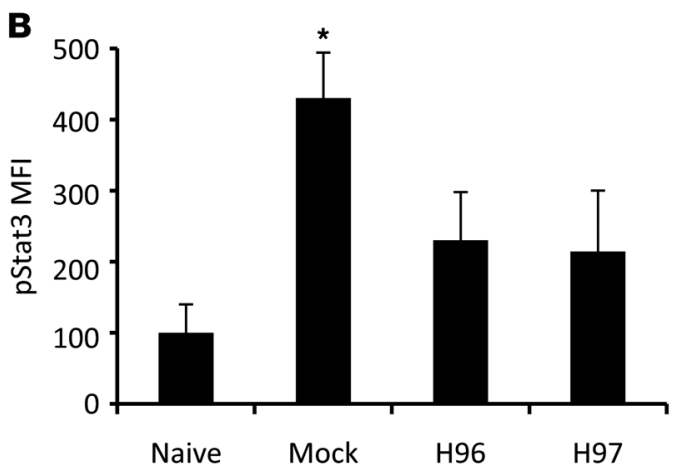

D

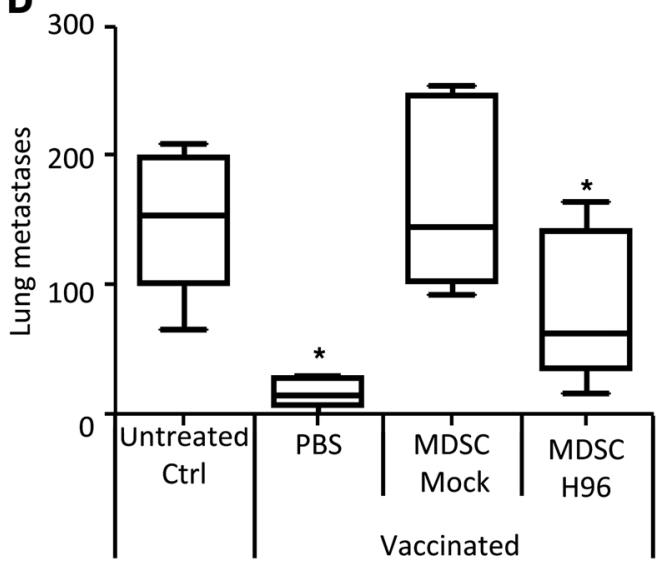

Figure 6

pStat3 expression in MDSCs is dependent on Hsp72 on TDEs. (A) Purified myeloid cells from naive WT or TLR2-deficient mice were either untreated or treated as indicated. (B) Naive mice were i.v. injected with TDEs from shRNA mock CT26 cells or Hsp72 shRNA CT26 clones H96 or H97. 18 hours later, spleen cells were harvested and pStat3 expression was determined by FACS. (C) Naive BALB/c mice were s.C. injected with $1 \times 10^{6} \mathrm{H} 96, \mathrm{H} 97$, or mock CT26 cells. 2 weeks later, spleens were harvested and pStat3 expression determined by FACS. For A-C, pStat3 was determined by FACS analysis on MDSC gated cells. Data represent MFI \pm SD. Experiments were performed in triplicate ( $n=3$ mice per group). Inset shows immunoblot of pStat3 expression in sorted MDSCs from mice bearing mock CT26 or Hsp72 shRNA CT26 clone H96. (D) Mice were vaccinated with frozen/thawed CT26 cells 1 week before i.v. injection of live CT26 cells admixed or not with MDSCs isolated from mice bearing shRNA mock-transfected or Hsp72 shRNA-transfected (clone H96) CT26 tumors. Twelve days later, lung metastasis numbers were evaluated. Experiments were performed in triplicate ( $n=5$ mice per group). For box and whisker plots, bottoms and tops of boxes show the 25th and 75 th percentiles, respectively, and middle bands show the median; whiskers show extrema. ${ }^{*} P<0.05$. Error bars represent mean + SD.

clude that the Hsp72/TLR2 pathway described here is a new pathway involved in MDSC activation. Hsp72 is a molecular chaperone present at elevated levels in various human tumors, and its expression often correlates with increased tumor cell proliferation, poor response to chemotherapy, and poor survival $(49,50)$. In addition to its classical intracellular localization, Hsp72 is also expressed on the plasma membrane of malignantly transformed cells or TDEs (51). Many reports also focused on the possible immunoadjuvant effect of Hsps, which enhance the maturation of dendritic cells (51) and NK cells (52), thus leading to immune-mediated protection (53). These results suggested that complexes of Hsps with peptides might represent a unique and efficient way to induce immunity, a hypothesis that was challenged by reports showing that Hsps can also downregulate an immune response in some autoimmune models such as rheumatoid arthritis in rats (54) and diabetes in mice (55). The mechanism proposed for the immunoregulatory function of Hsps involved the generation of IL-10-pro- ducing immunosuppressive T cells (56). In this regard, it is noteworthy that extracellular Hsp72 could also render APC resistant to adjuvant-induced maturation (57). In this study, we propose that Hsp72 expression in exosomes is detrimental for the antitumor response. To the best of our knowledge, this is the first study that provides a mechanistic explanation linking Hsp72 expression by tumor cells and the induction of immunosuppression in mice and humans. Hsp72 cytokine properties are well described and related to its capacity to bind to TLR4/CD14 complex (58) or TLR2 (59). Conflicting reports suggest that adjuvant Toll-dependent effects of Hsps are due to LPS or lipopeptide contamination (60). In our study, we performed physical measurement of the interaction between Hsp72 and TLR2 by surface plasmon resonance (Figure 5C). The in vivo immunosuppressive role of TDEs led us to search for drugs interfering with exosome secretion by tumor cells that might theoretically represent a strategy to restore tumor immunity and to impair tumor progression. A promising tool may be 

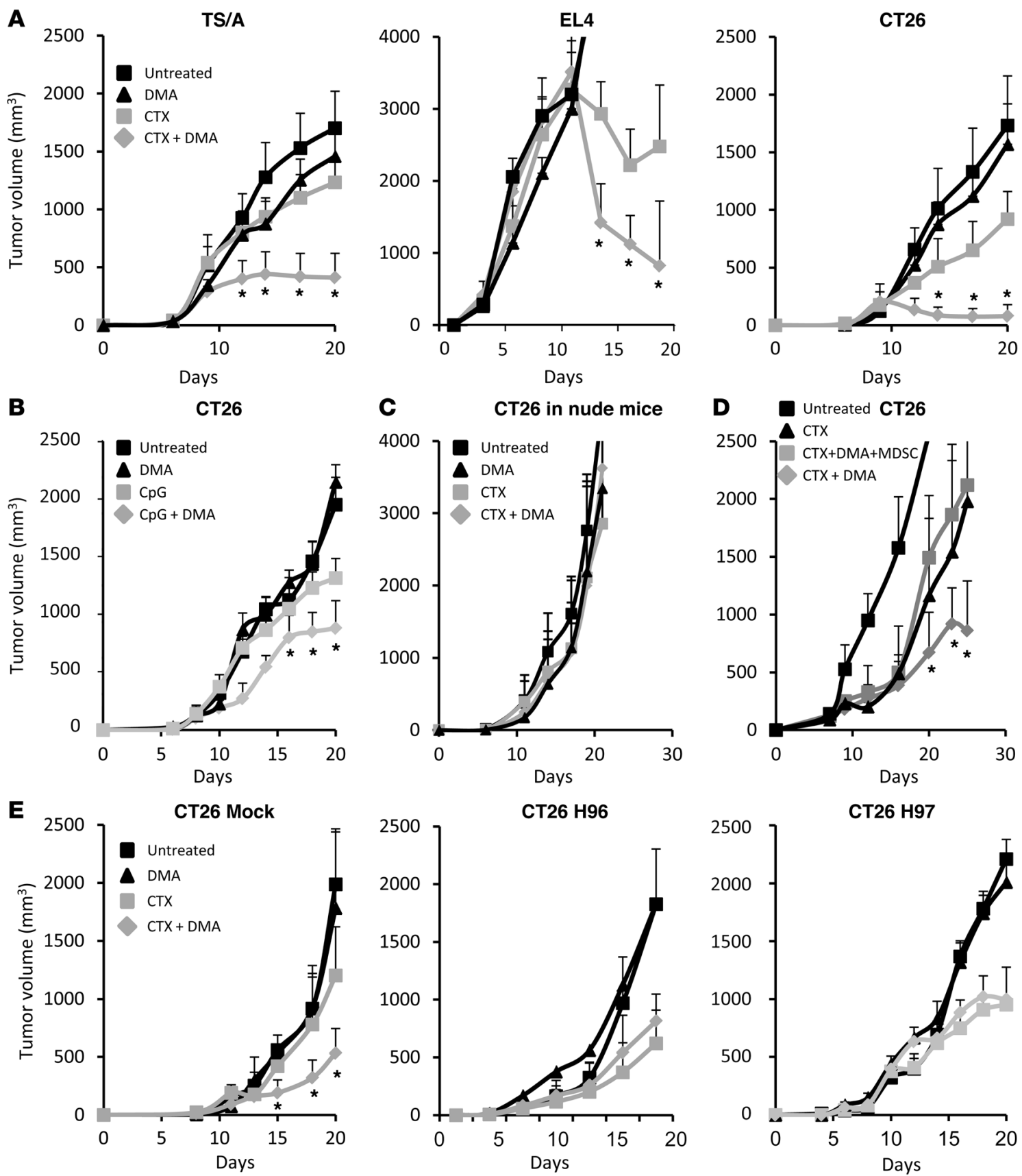

Figure 7

Exosome depletion restores the efficacy of immunotherapy. Immunocompetent mice (A) were injected s.c. with $1 \times 10^{6} \mathrm{CT} 26$, TS/A, or EL4 cells. Mice were then either untreated or treated with 1 i.p. injection of $100 \mathrm{mg} / \mathrm{kg}$ cyclophosphamide (CTX), daily i.p. injection of DMA, or both. Mean tumor volume $\pm \mathrm{SD}$ ( $n=6$ mice per group). (B) WT mice were injected as in A with CT26 and treated with intratumoral CpG 1668 injection (10 $\mu \mathrm{g}$ once a week) with or without DMA. (C) Nude mice were treated as in A. (D) BALB/c mice were injected s.c. with $1 \times 10^{6}$ CT26 cells. Mice were then either untreated or treated with 1 i.p. injection of cyclophosphamide or 1 i.p. injection of cyclophosphamide plus repeated daily i.p. injections of DMA with or without an i.v. adoptive transfer of $5 \times 10^{6} \mathrm{MDSC}$ from CT26 tumor-bearing mice. Data shown represent mean tumor volume \pm SD ( $n=5$ mice per group). (E) BALB/c mice were injected s.c. with $1 \times 10^{6}$ mock-transfected (left panel) or Hsp72 shRNA-transfected (clone H96, middle panel; clone H97, right panel) CT26 cells. Mice were then either untreated or treated with 1 i.p. injection of cyclophosphamide, i.p. injection of DMA twice a week, or both. Mean tumor volume $\pm \mathrm{SD}\left(n=6\right.$ mice per group). Experiments were performed in duplicate. ${ }^{*} P<0.05$.

represented by drugs such as DMA and its analog amiloride, which interfere with the activity of efflux pumps expressed on acidic vacuoles, such as $\mathrm{Na}+/ \mathrm{H}+$ export, and associated to exosome secretion (61). DMA and amiloride reduce exosome secretion in vitro and in vivo (Figure 3D) and blunt Stat3 phosphorylation in MDSCs and their $\mathrm{T}$ cell-suppressive function in mouse cancer models (Figure 3, F and $\mathrm{G}$ ) and in cancer patients (Figure 8, E and F). Moreover, in 3 mouse tumor models from 2 different mouse strains, DMA 

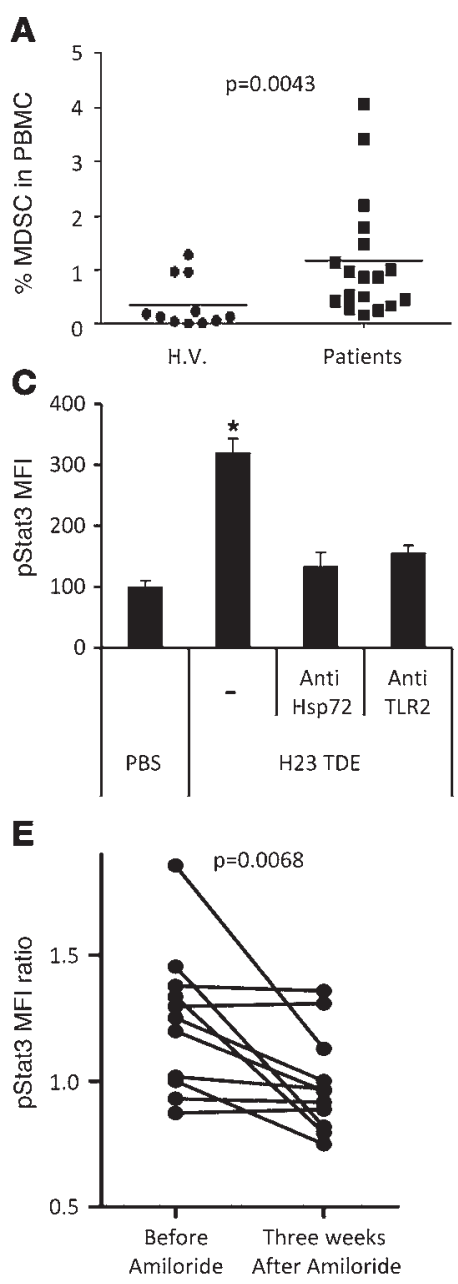
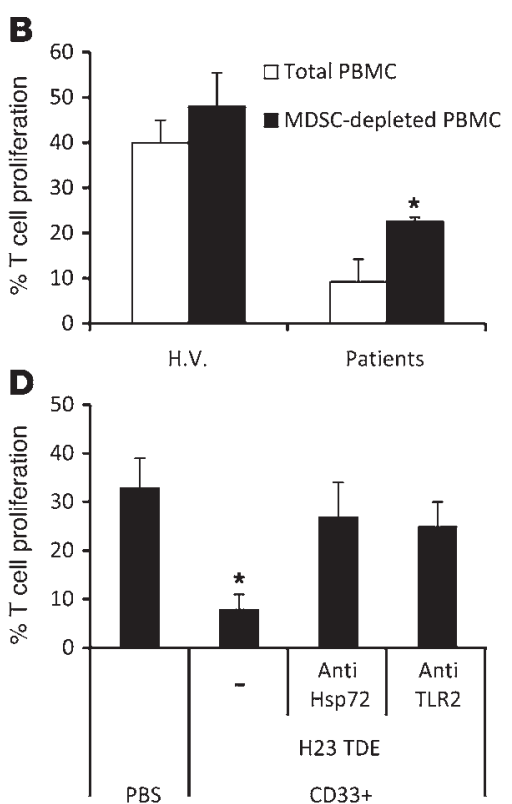

$\mathbf{F}$

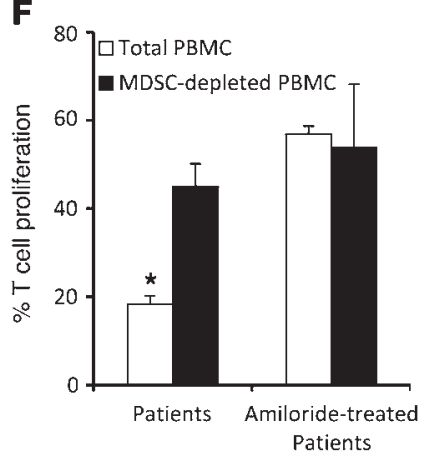

\section{Figure 8}

Exosomes produced by human cancer cell lines or metastatic cancer patients dictate Stat3 activation in MDSCs and their immunosuppressive function through TLR2 and Hsp72. (A) The frequency of MDSCs, defined as HLA-DR CD33+ cells, is shown in the PBMCs of healthy volunteers (H.V.) $(n=11)$ and metastatic cancer patients $(n=18)$. Each plot is an individual measure, and the horizontal bar is the mean. (B) Immunosuppressive function of MDSCs from peripheral blood of healthy volunteers and metastatic cancer patients on stimulated $\mathrm{T}$ cell proliferation. $\mathrm{T}$ cell stimulation was induced by a mixture of anti-CD2, anti-CD3, and anti-CD28 beads $(n=10)$. (C) PBMCs from healthy volunteers were cultured for 24 hours in medium alone or medium containing TDEs from $\mathrm{H} 23$ cells with or without blocking TLR2 Abs or anti-Hsp72 polyclonal Abs (pAbs). pStat3 was determined by flow cytometry on MDSC gated cells $(n=10)$. (D) Immunosuppressive function of MDSCs from blood of healthy volunteers either untreated or treated with TDEs from $\mathrm{H} 23$ cells alone or with blocking TLR2 Abs or anti-Hsp72 pAbs $(n=8)$. (E) PBMCs from metastatic cancer patients were incubated overnight in serum-free medium supplemented with autologous serum or PBS. pStat3 expression in gated MDSC was determined by flow cytometry. pStat3 MFI ratio between PBS and serum condition was represented. The same patients were sampled before and after 3 weeks of amiloride treatment $(n=11)$. (F) Immunosuppressive function of MDSCs prepared from peripheral blood of metastatic cancer patients, treated with amiloride or not treated, on $\mathrm{T}$ cell proliferation stimulated as in $\mathbf{B} .{ }^{*} P<0.05$. Error bars represent mean $+\mathrm{SD}$. enhances the antitumor efficacy of cyclophosphamide, an anticancer drug with cytotoxic and immunological properties (39).

In conclusion, we here describe an immunosuppressive pathway involved in tumor-induced tolerance in mice as well as in humans. This study supports the hypothesis that drugs interfering with exosome secretion such as amiloride may enhance the efficacy of current chemotherapies.

\section{Methods}

\section{Cell culture}

The mouse colon carcinoma CT26 cells, lymphoma EL4, embryo fibroblast $\mathrm{NIH} / 3 \mathrm{~T} 3$ cells, and human lung adenocarcinoma H23 were obtained from ATCC and mammary adenocarcinoma TS/A from Health Protection Agency Cultures Collections (HPACC). MSC cell lines were a gift from Vincenzo Bronte (Istituto Oncologico, Padova, Italy). All cells were grown in RPMI 1640 medium with glutamax-I (Lonza) supplemented with 10\% (v/v) FBS (Lonza) and with Pen/Strep Amphotericin B (Lonza) in an atmosphere of $95 \%$ air and $5 \% \mathrm{CO}_{2}$ at $37^{\circ} \mathrm{C}$. Absence of mycoplasma contamination was assayed every 2 weeks by PCR.

\section{Mice}

Female C57BL/6, BALB/c, and nude mice (aged 6 to 8 weeks) were obtained from the Centre d'élevage Janvier and from Charles River Laboratories. TLR2-/-, TLR4 $4^{-/}, \mathrm{MyD} 88^{-/-}$, and $T R I F^{-/-} \mathrm{C} 57 \mathrm{BL} / 6$ mice were provided by Bernhard Ryffel (CNRS UMR 6218, Orleans, France). These mice ere obtained from Shizuo Akira (Laboratory of Host Defense, Osaka, Japan) and crossed to C57BL/6 in UMR6218, Orleans, France. We obtained OT-1 TCR-transgenic mice (C57BL/6-Tg(TCR $\alpha \mathrm{TCR} \beta) 1100 \mathrm{mjb})$ from Christophe Borg (INSERM U645, Besancon, France).

To establish EL4, TS/A, and CT26 tumors, C57BL/6 and BALB/c mice were injected s.c. with $1 \times 10^{6} \mathrm{EL} 4, \mathrm{TS} / \mathrm{A}$, or CT26 cells, respectively. Tumor size was measured with calipers and is presented as the multiplication of the 2 longest dimensions. Some tumor-bearing mice were treated with daily i.p. injections of $1 \mu \mathrm{mol} / \mathrm{kg}$ DMA (Sigma-Aldrich) or $20 \mathrm{mg} / \mathrm{kg}$ omeprazole and 1 i.p. injection of $100 \mathrm{mg} / \mathrm{kg}$ cyclophosphamide (Sigma-Aldrich) when tumors were about $25 \mathrm{~mm}^{2}$. Some tumor-bearing mice were treated with weekly intratumoral injection of $0.5 \mathrm{mg} / \mathrm{kg} \mathrm{CpG}$ (InvivoGen).

For vaccination experiments, naive mice were injected in footpads with $1 \times 10^{6}$ frozen/thawed CT26 cells. These cells were warmed 10 minutes at $42^{\circ} \mathrm{C}$, then frozen in liquid nitrogen. One week after vaccination, mice were injected i.v. with $1 \times 10^{6}$ live CT26 cells, admixed or not with $5 \times 10^{6}$ MDSCs from DMA, PBS-treated tumor-bearing mice, MDSCs from 3-week-old shRNA mock CT26 tumor-bearing BALB/c mice or Hsp72 shRNA. After 12 days, lung metastasis number was determined. All animal experiments were approved by the local Animal Ethical Committee (Université de Bourgogne, Dijon, France).

\section{MDSCs}

Generation of cells from bone marrow progenitors. Bone marrow cells were obtained from the femurs and tibias of WT mice. Five million bone mar- 
row cells were cultured in RPMI 1640 medium supplemented with $10 \%$ FBS and $10 \mathrm{ng} / \mathrm{ml} \mathrm{GM-CSF}$ or in the presence of $50 \% \mathrm{v} / \mathrm{v}$ control (from 3T3 fibroblasts) or TCS, ultracentrifugated TCS (TDSFs), or exosomes (TDEs) $(50 \mu \mathrm{g} /$ well). On day 5 of culture, cells were collected and analyzed by flow cytometry.

Cell isolation and functional assays. Single-cell suspensions were prepared from spleens, and red cells were removed using ammonium chloride lysis buffer. Gr- $1^{+}$cells were isolated from spleens of tumor-bearing mice or naive mice by labeling the cells with PE Cy7 Ab to Gr-1, then using magnetic PE Cy7 beads and LS MACS columns (Miltenyi Biotec). CD11 $\mathrm{b}^{+}$ cells were isolated with anti-mouse CD11b beads (Miltenyi Biotech) according to the manufacturer's protocol. The $\mathrm{Gr} 1^{+}$cells were cultured 24 hours in different conditions: supplementation with $50 \%$ of control $3 \mathrm{~T} 3$ fibroblasts supernatant or TCSs, TDSFs, or TDEs (50 $\mu \mathrm{g} /$ well). Some cells were treated 24 hours or for indicated times with $1 \mu \mathrm{g} / \mathrm{ml} \mathrm{rHsp} 72$ (Stressgen ESP555), $10 \mathrm{ng} / \mathrm{ml}$ rIL-6 (R\&D Systems), $1 \mu \mathrm{g} / \mathrm{ml} \mathrm{Pam3CSK4}$ (InvivoGen), $0.25 \mu \mathrm{M}$ JSI124 (Calbiochem), $30 \mu \mathrm{M}$ STA21 (Biomol), $10 \mathrm{ng} / \mathrm{ml}$ LPS (Sigma-Aldrich), $50 \mu \mathrm{g} / \mathrm{ml}$ poly(I:C) (InvivoGen), $1 \mu \mathrm{g} / \mathrm{ml}$ anti-IL-6 mAb (R\&D Systems), or $5 \mu \mathrm{g} / \mathrm{ml}$ anti-Hsp72 pAb (Stressgen), $2 \mu \mathrm{g} / \mathrm{ml}$ anti-GM-CSF (Abcam).

Recombinant Hsp72 was tested for LPS contamination using Limulus amebocyte assay (Limulus amebocyte lysate QCL100; Cambrex). The level of endotoxin was less than the lowest control standard.

In functional assay, we used spleen cells from OT-1 mice. CD8 ${ }^{+} \mathrm{T}$ cells were purified with CD8 microbeads (Miltenyi Biotec) and stained 10 minutes with $\mathrm{CFSE}(1 \mu \mathrm{M}$ in PBS $)$ at $37^{\circ} \mathrm{C}$, then washed 3 times in complete medium. $\mathrm{CD} 8^{+}$ cells were cultured with specific peptide SIINFEKL (Bachem) $(10 \mu \mathrm{g} / \mathrm{ml})$ in the presence of MDSCs (1:1, 1:4, 1:8, 1:16 ratios). Three days later, proliferation of OT-1 cells was evaluated with CFSE dilution by flow cytometry.

Flow cytometry. For extracellular staining of immune markers, single-cell suspensions were prepared. Red cells were removed using ammonium chloride lysis buffer. We incubated $1 \times 10^{6}$ freshly prepared cells resuspended in RPMI $10 \%$ FBS with fluorochrome-coupled Abs to Gr1 and CD11b (eBioscience). Stat3-Alexa Fluor 488 and Ki67-FITC staining were carried out according to the manufacturer's protocol using the Cytofix/Cytoperm kit (BD Biosciences). All events were acquired by a BD Bioscience LSR-II device and analyzed with FlowJo (Tree Star). For IFN- $\gamma$ intracellular staining, spleens and tumors were harvested and dissociated as usual. Leukocytes were then cultured in vitro in 3 different conditions: coated anti-CD3 alone, coated anti-CD3 and killed tumor cells; and coated plus soluble antiCD3 $(2 \mu \mathrm{g} / \mathrm{ml})$, soluble anti-CD28 (2 $\mu \mathrm{g} / \mathrm{ml})$, and IL-2 (1000 UI/ml; Proleukin Chiron). Stimulation was maintained for 16 hours, with brefeldin A (GolgiPlug; BD) the last 4 hours. Cells were then harvested and stained for $\mathrm{CD} 4, \mathrm{CD} 8$, and intracellular IFN- $\gamma$ using the BD protocol.

Human MDSCs. Human MDSCs were isolated as follows: PBMCs were obtained from heparinized blood samples from either healthy volunteers or patients suffering from breast, colon, or prostate cancer (samples were obtained with informed consent and according to the local Ethical Committee CPP EST from Dijon, France) by centrifugation (800 $\mathrm{g}$ for 30 minutes) on a density cushion, using lymphocyte separation medium (Eurobio). MDSCs were analyzed by flow cytometry after labeling and characterized by the $\mathrm{CD}^{3} 3^{+} \mathrm{HLA}^{-} \mathrm{DR}-\mathrm{CD}^{-}$phenotype in the monocytic/ granulocytic morphometric gate. For Y705-pStat3 determination, PBMCs were incubated in AIM-V medium (GIBCO; Invitrogen) supplemented with components indicated in the figure legends for 18 hours. Then fixation and permeabilization were performed using BD Biosciences Phosflow standard protocol $\mathrm{N}^{\circ} \mathrm{III}$ for $\mathrm{pStat} 3$ level determination in the monocytic/granulocytic morphometric gate. The Abs used were purchased from BD Biosciences: anti-pY705-Stat3 PE conjugated (clone 4/P-STAT3), anti-human CD14 PE (M5E2), anti-human HLA-DR PerCP (L243 [G46-6]), anti-human
CD3 FITC (HIT3a), and anti-human CD33 APC (WM53). Some cells were treated 24 hours with $5 \mu \mathrm{g} / \mathrm{ml}$ anti-Hsp72 pAb (Stressgen) or $20 \mu \mathrm{g} / \mathrm{ml}$ anti-TLR2 pAb (Santa Cruz Biotechnology Inc.).

$\mathrm{T}$ cell proliferation was evaluated by CFSE dilution of CD33- ${ }^{-}$cells alone or with $\mathrm{CD}_{3}{ }^{+}$(in the original ratio) activated with the Treg Suppression Inspector (Miltenyi Biotec).

\section{Exosome isolation and labeling}

48-hour supernatants of confluent tumor cells were collected and sequentially centrifuged $\left(4^{\circ} \mathrm{C}\right)$ at $300 \mathrm{~g}$ for 10 minutes and then at $1200 \mathrm{~g}$ for 30 minutes. Exosomes were then pelleted at $100,000 \mathrm{~g}$ for 12 hours and washed once in PBS. The protein concentrations of exosomes were measured by Lowry assay (Bio-Rad). For some experiments, exosomes were further separated from other microparticles by centrifugation on a $30 \%$ sucrose/D20 cushion for 2 hours; these preparations were used to ascertain the presence of Hsp72 in the exosomal fraction and not in the other microparticles. For FACS staining, we bound the exosomes to surfactant-free white aldehyde-sulfate latex beads ( $3 \mu \mathrm{m}$; Invitrogen) and concentrated at $1 \times 10^{4} \mathrm{beads} / \mu \mathrm{l}$. We incubated $200 \mu \mathrm{g}$ TDEs with a $70 \mu \mathrm{l}$ bead suspension for 10 to 15 minutes and then for 1 hour at room temperature in a final volume of $1 \mathrm{ml}$ PBS. Beads were saturated for 30 minutes in glycine $(100 \mathrm{mM})$ at room temperature, then washed twice with PBS supplemented by $3 \%$ of FBS. A classic staining was realized on exosomes with control isotype or anti-Hsp72 (provided by Gabriele Multhoff, Department of Radiation Oncology, University Hospital, Technische Universität München) or antiHsc73 (Santa Cruz Biotechnology Inc.) Abs.

\section{Electronic microscopy}

Exosome preparations were stained with osmium tetroxide, then observed with a Hitachi H-7500 electronic microscope equipped with an AMT camera.

\section{Quantitation of released exosomes}

The amount of released exosomes was quantified by measuring the activity of acetylcholinesterase, an enzyme that is specifically present in these vesicles. Exosome fractions $(25 \mu \mathrm{l})$ were suspended in $100 \mu \mathrm{l}$ of PBS and

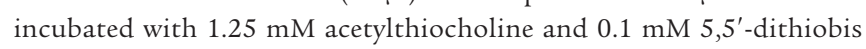
(2-nitrobenzoic acid) in a final volume of $1 \mathrm{ml}$. The change in absorbance at $412 \mathrm{~nm}$ was followed continuously. The data represent the enzymatic activity after 20 minutes of incubation.

\section{Molecular biology}

ELISA. ELISA kits were used to detect IL-6, IL-10 (BD Biosciences), VEGF, TNF- $\alpha$, PGE2 (R\&D Systems), and IL-1 $\beta$ (Biolegend) according to the manufacturers' protocol.

Western blotting. The following mouse mAbs were used: anti- $\beta$-actin (SigmaAldrich), anti-Hsc73, anti-Stat3 (Santa Cruz Biotechnology Inc.), antiHsp60 (Stressgen), and anti-flotillin (BD Biosciences). The following rabbit polyclonal Abs were used: anti-Hsp72, anti-Hsp90 $\beta$ (Stressgen), anti-versican, anti-HMGB1 (Abcam), anti-pStat3, anti-pErk, anti-Erk (Cell Signaling Technology), and anti-BSA (Sigma-Aldrich). Rat polyclonal anti-Grp94 (Stressgen) and goat polyclonal anti-tsg101 (Santa Cruz Biotechnology Inc.) were also used. Secondary Abs HRP-conjugated polyclonal goat anti-mouse, swine antirabbit, and goat anti-rat immunoglobulins (Jackson ImmunoResearch) and polyclonal rabbit anti-goat immunoglobulins (Dako) were also used.

\section{shRNA transduction}

CT26 cells were transduced with MISSION Lentiviral Transduction Particles (Sigma-Aldrich) to silence Hsp72 or with negative control particles according to the manufacturer's instructions. One population trans- 
duced with control particles (mock) and 5 transduced with 5 different shRNAs specific for Hsp72 (H95 to H99) were obtained, and 2 subclones (H96 and H97) were then selected after checking Hsp72 downregulation by Western blotting.

\section{siRNA transfection}

For in vivo experiments, Silencer Select Predesigned siRNA specific for murine IL-6 (forward: 5'-CUACCAAACUGGAUAUAAUtt-3', reverse: 5'-AUUAUAUCCAGUUUGGUAGca-3') or negative control siRNA (Applied Biosystems) were prepared with transfection reagent JetPEI (Polyplus Transfection) according to the manufacturer's instructions.

MDSCs were transfected in vitro with Silencer Select Predesigned siRNA specific for murine Stat3 (forward: 5'-GGGUGAAAUUGACCAGCAAtt-3', reverse: 5'-UUGCUGGUCAAUUUCACCCaa-3'; and forward: 5'-GAGUUGAAUUAUCAGCUUAtt-3', reverse: 5'-UAAGCUGAUAAUUCAACUCag- $3^{\prime}$ ) or negative control siRNA (Applied Biosystems) with transfection reagent JetPEI (Polyplus Transfection) according to the manufacturer's instructions.

CT26 was transfected in vitro with Silencer Select Predesigned siRNA specific for murine IL-6st (forward: 5'-GGAAAAUCUUGGAUUAUGAtt-3', reverse: 5'-UCAUAAUCCAAGAUUUUCCca-3') or negative control siRNA (Applied Biosystems) with transfection reagent INTERFERin (Polyplus Transfection) according to the manufacturer's instructions.

\section{SPR analysis}

Design and fabrication of homemade chips compatible with Surface Plasmon Resonance have been performed as previously published with the help of the MIMENTO technological platform (Besançon, France) (62). Biacore experiments were performed with the Biacore 2000 apparatus at $25^{\circ} \mathrm{C}$ with a flow rate of between 2 and $30 \mu \mathrm{l} / \mathrm{min}$.

\section{Statistics}

Results were analyzed with GraphPad Prism 5. Appropriated statistical tests were used according to the variance, matching pairs, and distribution. $P<0.05$ was considered significant. Data are represented as mean \pm SD.

\section{Acknowledgments}

This work was supported by special grants from the Fondation de France, the Association pour la Recherche sur le Cancer, the Institut National de la Santé et de la Recherche Médicale, and the Ligue Nationale Contre le Cancer (Region Bourgogne). F. Chalmin was supported by the Conseil Régional Bourgogne/INSERM, J. Vincent by the Institut National contre le Cancer. G. Mignot was funded by the Association pour la Recherche sur le Cancer. C. Garrido and E. Solary lead teams supported by the "Ligue Nationale Contre le Cancer." L. Apetoh is supported by EMBO. The authors thank the flow cytometry facility of IFR Santé-STIC.

Received for publication October 9, 2009, and accepted in revised form December 2, 2009.

Address correspondence to: François Ghiringhelli, Centre de Recherche, INSERM U866, Facultés de médecine et de Pharmacie, 7 Bd Jeanne d'Arc, 21079 Dijon, France. Phone: (33) 3-80-39-33-53. Fax: (33) 3-80-39-34-34; E-mail: fghiringhelli@cgfl.fr or francois. ghiringhelli@yahoo.fr.
1. Gabrilovich DI, et al. The terminology issue for myeloid-derived suppressor cells. Cancer Res. 2007;67(1):425; author reply 426

2. Ostrand-Rosenberg S, Sinha P. Myeloid-derived suppressor cells: linking inflammation and cancer. J Immunol. 2009;182(8):4499-4506.

3. Filipazzi P, et al. Identification of a new subset of myeloid suppressor cells in peripheral blood of melanoma patients with modulation by a granulocytemacrophage colony-stimulation factor-based antitumor vaccine. J Clin Oncol. 2007;25(18):2546-2553.

4. Mirza N, et al. All-trans-retinoic acid improves differentiation of myeloid cells and immune response in cancer patients. Cancer Res. 2006;66(18):9299-9307.

5. Srivastava MK, et al. Lung cancer patients' CD4(+) $\mathrm{T}$ cells are activated in vitro by MHC II cell-based vaccines despite the presence of myeloid-derived suppressor cells. Cancer Immunol Immunother. 2008;57(10):1493-1504.

6. Zea AH, et al. Arginase-producing myeloid suppressor cells in renal cell carcinoma patients: a mechanism of tumor evasion. Cancer Res. 2005;65(8):3044-3048.

7. Tu S, et al. Overexpression of interleukin-1beta induces gastric inflammation and cancer and mobilizes myeloid-derived suppressor cells in mice. Cancer Cell. 2008;14(5):408-419.

8 . Almand $B$, et al. Increased production of immature myeloid cells in cancer patients: a mechanism of immunosuppression in cancer. J Immunol. 2001;166(1):678-689.

9. Diaz-Montero CM, et al. Increased circulating myeloid-derived suppressor cells correlate with clinical cancer stage, metastatic tumor burden, and doxorubicin-cyclophosphamide chemotherapy. Cancer Immunol Immunother. 2009;58(1):49-59.

10. Ochoa AC, Zea AH, Hernandez C, Rodriguez PC. Arginase, prostaglandins, and myeloid-derived suppressor cells in renal cell carcinoma. Clin Cancer Res. 2007;13(2 Pt 2):721s-726s.

11. Nagaraj $S$, et al. Altered recognition of antigen is a mechanism of CD8+ T cell tolerance in cancer. Nat Med. 2007;13(7):828-835.

12. Gabrilovich DI, Nagaraj S. Myeloid-derived suppressor cells as regulators of the immune system. Nat Rev Immunol. 2009;9(3):162-174.

13. Nefedova Y, Fishman M, Sherman S, Wang X, Beg AA, Gabrilovich DI. Mechanism of all-trans retinoic acid effect on tumor-associated myeloid-derived suppressor cells. Cancer Res. 2007;67(22):11021-11028.

14. Nefedova Y, et al. Hyperactivation of STAT3 is involved in abnormal differentiation of dendritic cells in cancer. J Immunol. 2004;172(1):464-474.

15. Cheng $P$, et al. Inhibition of dendritic cell differentiation and accumulation of myeloid-derived suppressor cells in cancer is regulated by S100A9 protein. J Exp Med. 2008;205(10):2235-2249.

16. Corzo CA, et al. Mechanism regulating reactive oxygen species in tumor-induced myeloid-derived suppressor cells. J Immunol. 2009;182(9):5693-5701.

17. Wang $\mathrm{T}$, et al. Regulation of the innate and adaptive immune responses by Stat-3 signaling in tumor cells. Nat Med. 2004;10(1):48-54.

18. Lieblein JC, et al. STAT3 can be activated through paracrine signaling in breast epithelial cells. BMC Cancer. 2008;8:302.

19. Gallina G, et al. Tumors induce a subset of inflammatory monocytes with immunosuppressive activity on CD8+ T cells. J Clin Invest. 2006;116(10):2777-2790.

20. Kortylewski M, et al. Inhibiting Stat 3 signaling in the hematopoietic system elicits multicomponent antitumor immunity. Nat Med. 2005;11(12):1314-1321.

21. Mignot G, Roux S, Thery C, Segura E, Zitvogel L. Prospects for exosomes in immunotherapy of cancer. J Cell Mol Med. 2006;10(2):376-388.

22. Thery C, Zitvogel L, Amigorena S. Exosomes: composition, biogenesis and function. Nat Rev Immunol. 2002;2(8):569-579.

23. van Niel G, Porto-Carreiro I, Simoes S, Raposo G. Exosomes: a common pathway for a specialized function. J Biochem. 2006;140(1):13-21.

24 . Xiang $X$, et al. Induction of myeloid-derived sup- pressor cells by tumor exosomes. Int $J$ Cancer. 2009;124(11):2621-2633.

25 . Valenti R, et al. Human tumor-released microvesicles promote the differentiation of myeloid cells with transforming growth factor-beta-mediated suppressive activity on T lymphocytes. Cancer Res. 2006;66(18):9290-9298.

26. Zhong Z, Wen Z, Darnell JE Jr. Stat3: a STAT family member activated by tyrosine phosphorylation in response to epidermal growth factor and interleukin-6. Science. 1994;264(5155):95-98.

27. Chen SH, et al. Activated STAT3 is a mediator and biomarker of VEGF endothelial activation. Cancer Biol Ther. 2008;7(12):1994-2003.

28. Iero M, et al. Tumour-released exosomes and their implications in cancer immunity. Cell Death Differ. 2008;15(1):80-88.

29. Savina A, Furlan M, Vidal M, Colombo MI. Exosome release is regulated by a calcium-dependent mechanism in K562 cells. J Biol Chem. 2003;278(22):20083-20090.

30. Leadbetter EA, et al. Chromatin-IgG complexes activate $B$ cells by dual engagement of IgM and Toll-like receptors. Nature. 2002;416(6881):603-607.

31. Apetoh L, et al. Toll-like receptor 4-dependent contribution of the immune system to anticancer chemotherapy and radiotherapy. Nat Med. 2007;13(9):1050-1059.

32. Kim S, et al. Carcinoma-produced factors activate myeloid cells through TLR2 to stimulate metastasis. Nature. 2009;457(7225):102-106.

33. Tsan MF, Gao B. Endogenous ligands of Toll-like receptors. J Lenkoc Biol. 2004;76(3):514-519.

34. Vabulas RM, et al. Endocytosed HSP60s use toll-like receptor 2 (TLR2) and TLR4 to activate the toll/interleukin-1 receptor signaling pathway in innate immune cells. J Biol Chem. 2001;276(33):31332-31339.

35. Asea A, et al. Novel signal transduction pathway utilized by extracellular HSP70: role of toll-like receptor (TLR) 2 and TLR4. J Biol Chem. 
2002;277(17):15028-15034

36. Vabulas RM, et al. HSP70 as endogenous stimulus of the Toll/interleukin-1 receptor signal pathway. J Biol Chem. 2002;277(17):15107-15112.

37. Vabulas RM, et al. The endoplasmic reticulum-resident heat shock protein Gp96 activates dendritic cells via the Toll-like receptor $2 / 4$ pathway. J Biol Chem. 2002;277(23):20847-20853.

38. Park JS, et al. Involvement of toll-like receptors 2 and 4 in cellular activation by high mobility group box 1 protein. J Biol Chem. 2004;279(9):7370-7377.

39. Ghiringhelli $\mathrm{F}$, et al. CD4+CD25+ regulatory $\mathrm{T}$ cells suppress tumor immunity but are sensitive to cyclophosphamide which allows immunotherapy of established tumors to be curative. Eur Immunol. 2004;34(2):336-344.

40. Roux S, et al. CD4CD25 Tregs control the TRAILdependent cytotoxicity of tumor-infiltrating DCs in rodent models of colon cancer. J Clin Invest. 2008;118(11):3751-3761.

41. Mandruzzato S, et al. IL4Ralpha+ myeloid-derived suppressor cell expansion in cancer patients. J Immunol. 2009;182(10):6562-6568.

42. Pan PY, et al. Reversion of immune tolerance in advanced malignancy: modulation of myeloid-derived suppressor cell development by blockade of stem-cell factor function. Blood. 2008;111(1):219-228.

43. Ozao-Choy J, et al. The novel role of tyrosine kinase inhibitor in the reversal of immune suppression and modulation of tumor microenvironment for immune-based cancer therapies. Cancer Res. 2009;69(6):2514-2522.

44. Sinha P, Clements VK, Fulton AM, Ostrand-Rosen- berg S. Prostaglandin E2 promotes tumor progression by inducing myeloid-derived suppressor cells. Cancer Res. 2007;67(9):4507-4513.

45. Andre F, et al. Malignant effusions and immunogenic tumour-derived exosomes. Lancet. 2002;360(9329):295-305.

46. Wolfers J, et al. Tumor-derived exosomes are a source of shared tumor rejection antigens for CTL cross-priming. Nat Med. 2001;7(3):297-303.

47. Abusamra AJ, et al. Tumor exosomes expressing Fas ligand mediate CD8+ T-cell apoptosis. Blood Cells Mol Dis. 2005;35(2):169-173.

48. Youn JI, Nagaraj S, Collazo M, Gabrilovich DI. Subsets of myeloid-derived suppressor cells in tumorbearing mice. J Immunol. 2008;181(8):5791-5802.

49. Jaattela M. Escaping cell death: survival proteins in cancer. Exp Cell Res. 1999;248(1):30-43.

50. Calderwood SK, Khaleque MA, Sawyer DB, Ciocca DR. Heat shock proteins in cancer: chaperones of tumorigenesis. Trends Biochem Sci. 2006; 31(3):164-172.

51. Singh-Jasuja $\mathrm{H}$, et al. The heat shock protein gp96 induces maturation of dendritic cells and down-regulation of its receptor. Eur J Immunol. 2000;30(8):2211-2215.

52. Multhoff G. Activation of natural killer cells by heat shock protein 70 . Int $J$ Hyperthermia. 2002;18(6):576-585.

53. Millar DG, et al. Hsp70 promotes antigen-presenting cell function and converts T-cell tolerance to autoimmunity in vivo. Nat Med. 2003 ; 9(12):1469-1476.

54. Kingston AE, Hicks CA, Colston MJ, Billingham ME. A 71-kD heat shock protein (hsp) from Myco- bacterium tuberculosis has modulatory effects on experimental rat arthritis. Clin Exp Immunol. 1996;103(1):77-82.

55. Elias D, Markovits D, Reshef T, van der Zee R, Cohen IR. Induction and therapy of autoimmune diabetes in the non-obese diabetic (NOD/Lt) mouse by a $65-\mathrm{kDa}$ heat shock protein. Proc Natl Acad Sci US A. 1990;87(4):1576-1580.

56. Prakken BJ, et al. Induction of IL-10 and inhibition of experimental arthritis are specific features of microbial heat shock proteins that are absent for other evolutionarily conserved immunodominant proteins. J Immunol. 2001;167(8):4147-4153.

57. Aneja R, Odoms K, Dunsmore K, Shanley TP, Wong HR. Extracellular heat shock protein-70 induces endotoxin tolerance in THP-1 cells. J Immunol. 2006;177(10):7184-7192.

58. Asea A, et al. HSP70 stimulates cytokine production through a CD14-dependant pathway, demonstrating its dual role as a chaperone and cytokine. Nat Med. 2000;6(4):435-442.

59. Galloway E, et al. Activation of hepatocytes by extracellular heat shock protein 72. Am J Physiol Cell Physiol. 2008;295(2):C514-C520.

60. Tsan MF, Gao B. Heat shock proteins and immune system. J Leukoc Biol. 2009;85(6):905-910.

61. Gupta S, Knowlton AA. HSP60 trafficking in adult cardiac myocytes: role of the exosomal pathway. AmJ Physiol Heart Circ Physiol. 2007;292(6):H3052-H3056.

62. Boireau W, Rouleau A, Lucchi G, Ducoroy P. Revisited BIA-MS combination: entire "on-a-chip" processing leading to the proteins identification at low femtomole to sub-femtomole levels. Biosens Bioelectron. 2009;24(5):1121-1127. 